\title{
Electrical Signal Path Study and Component Assay for the MAJorana N-Type Segmented Contact Detector
}

First version 24 Aug. 2008, revised 24 Feb. 2009

M. Amman, M. Bergevin, Y.D. Chan, J.A. Detwiler, B.K. Fujikawa, K.T.Lesko, P.N. Luke, G. Prior, A.W. Poon, A.R. Smith, K. Vetter, H. Yaver, and S. Zimmermann

Lawrence Berekley National Laboratory

This work is supported by the U.S. Department of Energy

DOE/LBNL contract number DE-AC02-05CH11231, DE-AC03-76SF00098 


\section{Contents}

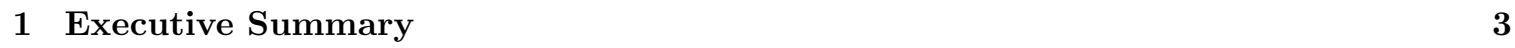

\begin{tabular}{|ll|}
\hline Introduction & $\mathbf{5}$ \\
\hline
\end{tabular}

2.1 The NSC Detector Concept . . . . . . . . . . . . . . . . . . . . . . . . . . . 5

2.2 Scope of the R\&D Activity . . . . . . . . . . . . . . . . . . . . . . . . . . 6

3 Cable and Signal Study $\quad 6$

3.1 Baseline Electrical Configuration and Detector Geometry for Signal Path Modeling . 6

3.2 Low Mass Cable Simulation Study . . . . . . . . . . . . . . . . . . . 7

3.2.1 Dimensions and Capacitance Matrices for the Cables . . . . . . . . . . . . . . 8

3.2 .2 The Testbed Prototype Cable . . . . . . . . . . . . . . . . . . . . . . . 10

3.3 Measurements Without a Detector . . . . . . . . . . . . . . . . . . . . . . . 11

3.3 .1 Risetime Measurements . . . . . . . . . . . . . . . . . . . . . . . . . . . . . . 12

3.3 .2 Crosstalk Measurements . . . . . . . . . . . . . . . . . . . . . . . . . . . . 13

3.3 .3 Noise Measurements . . . . . . . . . . . . . . . . . . . . . . . . 14

3.4 Measurements with a Segmented Ge Detector . . . . . . . . . . . . . . . . . . . . . 15

3.4.1 Energy Resolution . . . . . . . . . . . . . . . . . . . . . 17

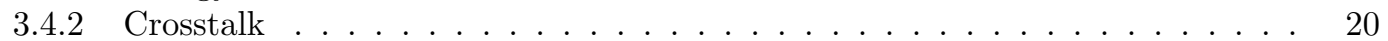

3.5 SEGA Electronics Support . . . . . . . . . . . . . . . . . . . . . . . . . . 20

4 Alternative Capacitive Coupling for the Central Contact Readeout 24

5 Material Screening Related to the Signal Path 28

5.1 Substrate Material for the LMFE . . . . . . . . . . . . . . . . . . . . . . . . . . . . . 28

5.2 New candidate material for cables - Parylene INAA at MNRC. . . . . . . . . . . . . 28

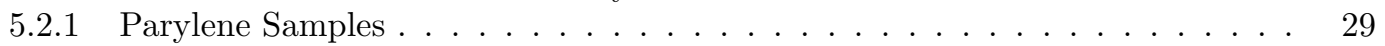

5.2 .2 Ceramic Standard . . . . . . . . . . . . . . . . . . . . . . . 29

5.2 .3 Irradiation at MNRC . . . . . . . . . . . . . . . . . . . . . . . 29

5.2 .4 Sample Transfer . . . . . . . . . . . . . . . . . . . . . . . . . . . . . . 29

5.3 Counting and Results . . . . . . . . . . . . . . . . . . . . . . . . . . . 31

5.3 .1 Potential Improvements . . . . . . . . . . . . . . . . . . . . . . . . 33

5.4 Other samples. . . . . . . . . . . . . . . . . . . . . . . . . 33

6 Summary and Conclusion $\quad 34$

6.1 Recommendation for the Segment Readout . . . . . . . . . . . . . . . . . . . . . . . 34

6.2 Recommendation for the Central Contact . . . . . . . . . . . . . . . . . . . . . . . . 34 


\section{Executive Summary}

The purpose of the present $R \& D$ task is to explore various issues related to the deployment of highly segmented low-background Ge detectors in the Majorana experiment 11. A significant challenge involved in deploying an array of these NSC (N-type Segmented Contact) detectors is to simultaneously satisfy competing requirements for the mechanical design, electrical readout performance, and radiopurity specifications. In order to minimize radioactivity originating from the electronics components, it is desirable to position the electronics outside of the detector cryostat, including the important first FET stage. However, this will force the raw pulses to pass through an atypically long cable length, potentially affecting the quality of the final signal. The NSC detectors could also have up to 37-channels of electronics (1 central contact plus 6x6 segments), so signal crosstalk problems need to be studied. The central contact of the NSC detectors will be operated in AC-coupling mode, which conventionally requires the presence of a large size high voltage decoupling capacitor close to the germanium crystal; having such massive, potentially dirty components near to the detectors is not desirable for Majorana. Common to all rare search double-beta decay experiments, there is a very stringent requirement on the acceptable radioactivity level of all the components that would be present. Therefore material selection and radioactivity screening is necessary.

The R\&D task was conducted at the Lawrence Berkeley National Laboratory (LBNL) by the Neutrino Astrophysics Group in cooperation with staff from the Engineering Division (S. Zimmerman, H. Yaver, M. Amman, and P.N. Luke) and the Low Background Facility (A. Smith), from Nov 2007 - Oct 2008. The goal was to obtain viable solutions for the above mentioned problems based on design, simulation, prototyping, and measurement. The specific NSC-related R\&D tasks listed in the MAJORANA DUSEL R\&D Proposal include:

- Mechanical design for NSC detectors.

- Electronics readout design for NSC detectors.

- Modification and initial demonstration of design with the GRETINA Prototype II (GP2) detector.

In addition to these, a small amount of LBNL engineer time was dedicated for electrical readout design for the SEGA detector.

A critical component of the original NSC design plan was to acquire funding for a functional $6 \times 6$ N-type segmented detector for in-situ signal studies. This crystal would preferably have had the same geometry as the final NSC detector since any optimized signal path solution will be quite complex (prone to cross-talk, microphonics, etc.) and therefore could be strongly coupled to the geometry of the detector. Although the received funding was not sufficient to purchase such a prototype detector, the GRETINA collaboration graciously lent us an old prototype segmented Ge detector (GP2) for the purposes of this R\&D, as indicated in the third bullet above. Unfortunately, the GP2 detector has a substantially different geometry from that envisioned for MAJORANA (encapsulated and tapered hexagonal towards the front rather than open and cylindrical in shape), and suffered from significant performance degradation and instability (see Section 3.4). Best effort had been attempted to make use of this detector for our R\&D task but there were limitations with respect to the originally proposed scope.

Prior to this R\&D task, LBNL staff initiated a mechanical design concept for the NSC mount design and presented it to the MAJORANA Collaboration [2]. However, due to the lack of additional funding support for local mechanical engineers, most of the subsequent NSC mechanical design work was shifted to designers at CENPA, University of Washington, in an cooperative effort. LBNL staff continued contributing in a consulting and advisory capacity to the design task.

Given these constraints, we chose to focus our resources on the design of the electrical readout, and on radiopurity issues related to the electrical and mechanical designs. The main tasks carried out included the following: 


\section{Signal study and optimization of the warm FET electronics option for the segment readout}

The signal cable length between the crystal and the warm FET is estimated to be about 30 inches long. A simulation of signal transmission in such a long cable was performed and prototype signal cables based on that study were designed and fabricated. The cable and preamplifiers were then connected to the GP2 detector, and the actual performance of the cable and preamplifier system for this particular configuration was studied by carrying out gamma source measurements.

\section{Alternative AC-coupling method and a Low Mass Front End (LMFE) board for the central contact readout}

Traditionally a fairly large size capacitor is used to decouple the signal from the high voltage bias. This is not desirable for MAJORANA as it could introduce a high level of radioactive backgrounds. We have taken an alternative AC-coupling approach so that the required capacitance could be drastically reduced to the point that all the front-end components (including the decoupling capacitor) could be fabricated onto a very small-mass circuit board. The $R \& D$ in this area was successful and a small-footprint prototype board was fabricated and tested.

\section{Assaying potential candidate materials for cables and components}

Part of the available funding was spent performing material radioactivity screening, including the application of neutron activation techniques followed by direct gamma counting at the Lab's Low Background Facility.

Part (1) of this work was affected by the severe instability of the GP2 detector (see Section 3.4), and only a fairly limited comparison with data could be performed. The repair cost of the detector was very high and not possible within the funds allocated to the present work. It was found that the long cable behaves well on the bench with a simulated input capacitance (matched to those from the GP2 detector, which has both cold- and warm-FET segments), but when connected via a large and well-shielded cable box to the detector, measurement shows worse performance than expected from simulation predictions. This discrepancy was traced down to the coupling of the signal to the large bench-top signal box that holds the cable, and that the signal connection to the GP2 was limited by its existing hardware design. Due to the strongly coupled nature of a closelypacked multi-segment detector system like GP2, it was evident that the measured results depended sensitively on the external connection method of the cables, which was not optimized in the present case. Nevertheless, we were able to conclude that the warm FET plus long signal cable configuration will function well in terms of getting a robust handle on gamma interaction site multiplicity (number of segments hit). It remains a viable option for reconstruction of vertices, but a more detailed in-situ study would need to be carried out to assess the ultimate performance of the proposed configuration. The effect of the long cable on the cold FET channels was determined to be relatively small. Further, the GP2 warm-readout scheme was deemed sufficient for the needs of the SEGA detector. LBNL engineers leveraged this work to produced an improved preamplification stage for the SEGA test cryostat.

Part (2) of this work was completed quite successfully with the fabrication and testing of a prototype low-mass front end board (see Section 4). The noise performance of this board is very good even at room temperature, but there are small intermittent discharge pulses observed when HV bias is applied. A further optimized choice of material and/or a board geometry change could potentially improve the situation. A similar board based on a different substrate is planned but is outside the scope of the present task. As a by-product, similar boards but with a simpler dc-coupling scheme (less components) on a different substrate material could also be fabricated.

Tasks in part (3) include the radioactivity screening of candidate cables, substrate material, and assembled preamplifier units, as well as performing Neutron Activation Analysis (NAA) of Parylene which could be used for cable fabrication (Section 5). 


\section{Introduction}

Whether neutrinos are Majorana particles or not is among the most fundamental physics questions to be answered today [1. Observing the neutrinoless nuclear double-beta decay process is the only known experimental technique to probe this important problem.

${ }^{76} \mathrm{Ge}$ is a well-known double-beta decay nucleus around which several generations of experiments have been designed and performed during the last couple of decades. The first generation of $\mathrm{Ge}$ experiments consisted of a single non-enriched HPGe crystal mounted in a low-radioactivity cryostat and readout with standard spectroscopic electronics. The second generation of experiments, such as the LBL/UCSB experiment in Oroville, California [3, employed multi-detectors assemblies with aggressive material selection (such as silicon-based cold fingers and copper electroplating technology for electronics parts) as well as elaborate active veto provisions. The third generation of experiments, such as the Heidelberg-Moscow [4] and IGEX [5] experiments, employing improved low-background techniques and materials including front-end electronics components, deployed crystals enriched in ${ }^{76} \mathrm{Ge}$ to increase the source mass drastically. A positive observation of the double-beta decay peak was reported by one of these groups 4, but that conclusion has remained controversial. At the heart of the controversy is the fact that these third-generation experiments relied on traditional "single signal" readout methods, which have relatively weak discrimination and diagnostic power once the final data set is obtained.

MAJORANA [1] and GERDA [6] represent the fourth generation of Ge double-beta decay experiments. The goal of these new projects is to obtain a definitive result by mounting a much larger scale experiment (e.g., with a much larger ${ }^{76} \mathrm{Ge}$ mass), and by employing low background fabrication techniques and more powerful detector technologies with advanced analytic capabilities.

\subsection{The NSC Detector Concept}

A major improvement for MAJORANA over the previous generations of experiments is the availability of more advanced Ge detector technologies, such as N-type Segmented-Contact (NSC) germanium detectors [6]. Unlike a conventional HPGe detector, where the the signal is readout only from the central electrode (full volume) of the crystal, a segmented detector has multiple electrodes at the outer detector surface. This electrode configuration provides independent charge collection from different subvolumes within the crystal, giving robust information about the number of gamma interaction sites inside the crystal that is not prone to pulse-shape instabilities that could affect the performance of other detector types. The pulse shapes captured on the different electrodes provides additionally a measure of the spatial locations of the interaction sites. Using such additional analytic capabilities to reject multiple interaction events and measure their spatial distribution can improve drastically the discriminating power of the experiment, producing more sensitive, definitive and convincing results.

Even though the segmented HPGe technology is relatively well established and has been deployed successfully in several large scale nuclear spectroscopic projects such as GRETINA [7 and AGATA [8], there are challenges when applied to MAJORANA, primarily due to the ultra low radioactivity background requirement for rare search experiments of this type. Of particular concern is the overall radioactivity of the internal hardware and electrical components in the immediate vicinity of the detector. These detectors require a large number of segment signals as well as a large accoupling capacitor to decouple the central electrode signal from the high voltage bias. Even if they can be fabricated cleaning, simply handling the large number of components in a low background environment will also pose a significant challenge. These components must be implemented without compromising the ultra-low radioactivity requirements for a sensitive rare-event search. 


\subsection{Scope of the R\&D Activity}

In order to suppress extra radioactivity contributions due to the electrical components one has to not only minimize the mass and radioimpurity of the parts used in the signal readout circuitry, but also position them as far away from the crystal as possible. This requirement can be more easily met by putting the large number of FETs, front-end components, and preamplifier electronics for the segment channels outside of the detector cryostat. This so-called "warm-FET" configuration is possible only if the impacts on signal quality and crosstalk for such a multi-channel system are acceptable.

One should also keep in mind that while the basic low activity radioactivity requirements are the same, performance-wise there are subtle differences between the requirements for the full volume (FV) central contact and the segment signals. The FV signal contains the full-energy information of the event, so for that contact it is preferable to optimize for best possible energy resolution with the cold FET located as close as possible inside the cryostat. The segment signals can be optimized for position and multiplicity information only, since their energy information is not used extensively. A fast-rising signal, with minimal parts count and low-power-consumption preamps are preferred.

The circuitry design of analogue preamps is a well understood and relatively standard procedure. Many spectroscopic preamplifier designs, fine-tuned for Ge detectors, already exist in the commercial sector, in collaborations such as GRETINA and AGATA, and in instrumentation groups at LBNL and elsewhere. Therefore, except for the low-radioactivity requirement, we assume that the preamplifier is not a major issue for the signal path design.

Consequently, we have identified three critical areas for R\&D for meeting the low-radioactivity and performance requirements for the FV and segment signal paths. Since the FV signal for a N-Type segmented detector has to be AC-coupled, reducing the radioactivity contribution from the extra coupling capacitor is essential. For this we have explored the option of reducing the required capacitance by using a non-standard circuit topology (Section 4). For the segment signals, while it is a common practice to run the segment channel FETs warm (outside the vacuum cryostat) as is done, for example, in GRETINA, it is desirable to maximize the distance between the components and the crystals. Therefore it is crucial to understand and optimize signal transmission associated with long cable paths $(\sim 0.8$ meter), and find favorable operating conditions with respect to cable induced noise, rise-time degradation, and signal cross-talk etc (Section 31). Finally, to address component radiopurity, we included as a component of our R\&D the radioactivity screening and NAA of candidate cables, substrate materials, and assembled preamplifier units (Section 5).

\section{Cable and Signal Study}

The objectives of this part of the R\&D were to optimize the charge sensitive preamplifiers and the connection cables between the Ge crystal and the output of the preamp for both central and segment contacts.

The parameters we targeted for optimization in this signal R\&D were the following:

- Crosstalk: compromise between cable length, nominal configuration, materials, mass, etc.

- Amplifier noise: capacitance, rise time, cable length, stability factors, component selection

- Rise time, bandwidth and stability (required for signal decomposition and reconstruction)

- Microphonics and grounding options

\subsection{Baseline Electrical Configuration and Detector Geometry for Signal Path Modeling}

We have to assume a baseline configuration for the electronics as well as the physical geometry of the detector array in order to proceed with simulation and optimization. In particular, this assumption 


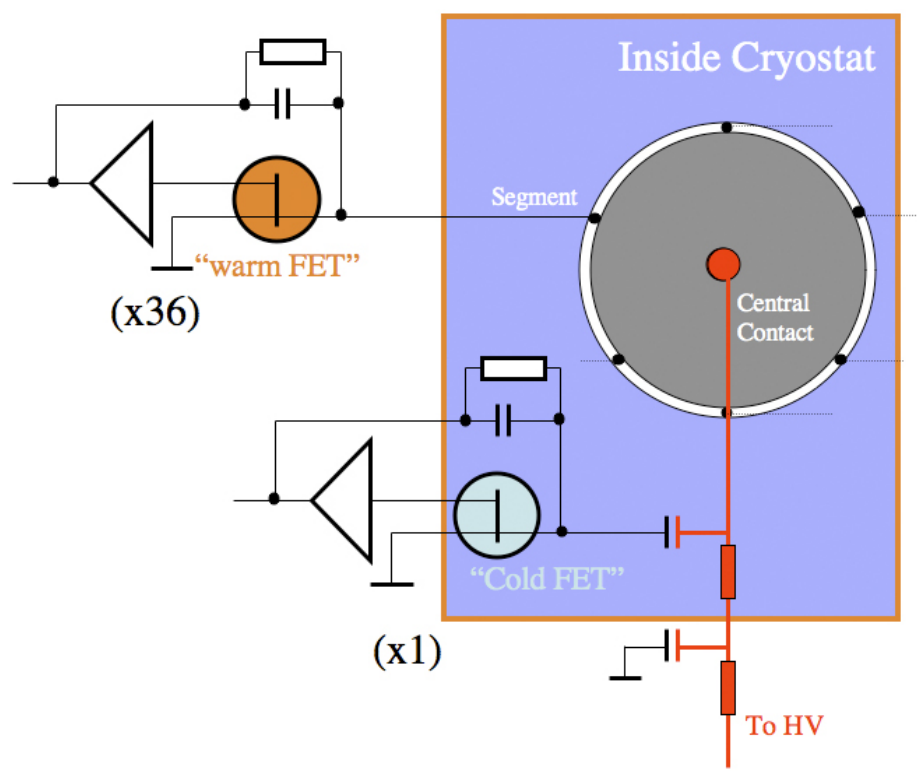

Figure 1: Baseline cold-warm hybrid electronics configuration for this work.

will determine the total length of the multi-trace cables we have to deal with. We have consequently adopted the hybrid cold-warm FET electronics approach (Fig. 1) based on experiences drawn from other large Ge-array systems such as GRETINA. The physical geometries of the detector and mount were derived from a candidate NSC Module conceptual design developed within the MAJORANA Collaboration (Fig. 2).

The baseline configuration of electrical components for the segments and central contact of the detector are therefore as follows:

- Segment: Put warm FET and feedback components in a miniature board about 30-inches away from the crystal, and outside the vacuum cryostat. It is possible that the complete preamplifier is also assembled in this board.

- Central contact: Using standard cold FET and feedback components configuration, close to the crystal and inside the vacuum cryostat. The rest of the amplifier is outside the vacuum, could be close to where the segment warm FETs are located.

\subsection{Low Mass Cable Simulation Study}

In order to avoid mass and heat close to the Ge crystal, we are proposing to move the segment FETs and the remaining of the segment amplifiers farther from the crystal. We will need then a low mass and low capacitance cable to connect the input FETs with the segments.

To decrease the crosstalk between the traces on the cable, one would prefer to layout the traces as far apart as reasonable. However, as Figs. 2 and 3 show, there will be limited width to pass the cable from the crystal segment and the top of the flange (the cable is depicted in blue in Fig. 3), which in turn will limit the spacing between the traces. The proposal is to assemble this cable behind the support structure to reduce background on the crystal and also to provide a ground reference for the signals along the cable. Therefore, the optimum approach is to consider two cable layouts: a shorter front-end cable between the segment and the top of the flange, and the other 


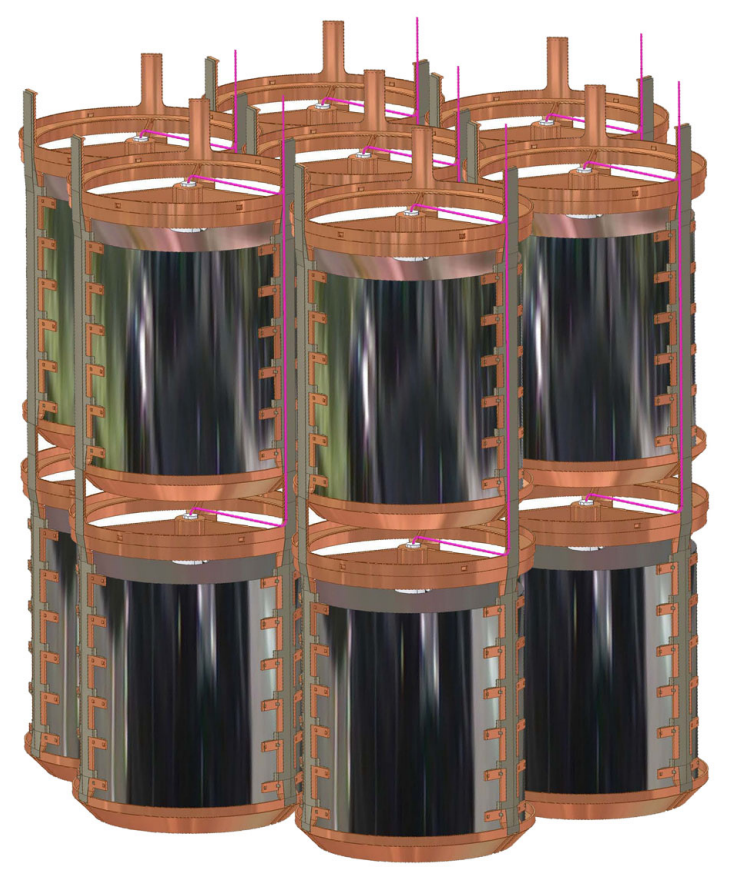

Figure 2: Engineering rendition of a candidate design for the NSC Module

longer one (signal transfer cable), which is not shown in Fig. 3 , from the flange to the vacuum-to-air feed-through.

We have performed simulations to estimate the behavior of both the short and long cables when connected to charge input amplifiers. We have used the HyperLynx software package to estimate the characteristics of the cables (capacitance, inductance, etc.) and SPICE to simulate the signal behavior (including crosstalk) when several segments are connected to the cables and amplifiers, with realistic preamplifier circuits. An example is shown in Fig. 4 . From these simulations we concluded that for the signals of interest the main form of crosstalk is due to capacitive coupling and that using a ground trace between the signals traces reduces this coupling significantly. For the final cable design this will require 24 traces, 12 for signals (for a total of 12 segments, see Fig. 3) and 12 for ground. Also, decreasing the thickness of the substrate (in this case Kapton) and avoiding coverlay further reduce the capacitive coupling. Fig. 5 shows the relationship between cable geometry optimization and the reduction of crosstalk and capacitance to ground (substrate is Kapton, with dielectric constant of 3.9). The actual dimensions of the two cables are shown below. We selected these dimensions because they are within the normal fabrication capacity of the industry. For example, it is difficult to manufacture a $1 \mathrm{~m}$ long cable with $50 \mu \mathrm{m}$ traces, and this is the reason we selected $100 \mu \mathrm{m}$ for the long cable.

\subsubsection{Dimensions and Capacitance Matrices for the Cables}

Here are the optimized results we obtained for the short cable (the width of the cable is limited by the crystal-mount support structure, see Fig. 22):

- Width: $\sim 1 \mathrm{~cm}$

- Trace thickness: $5 \mu \mathrm{m}$ 


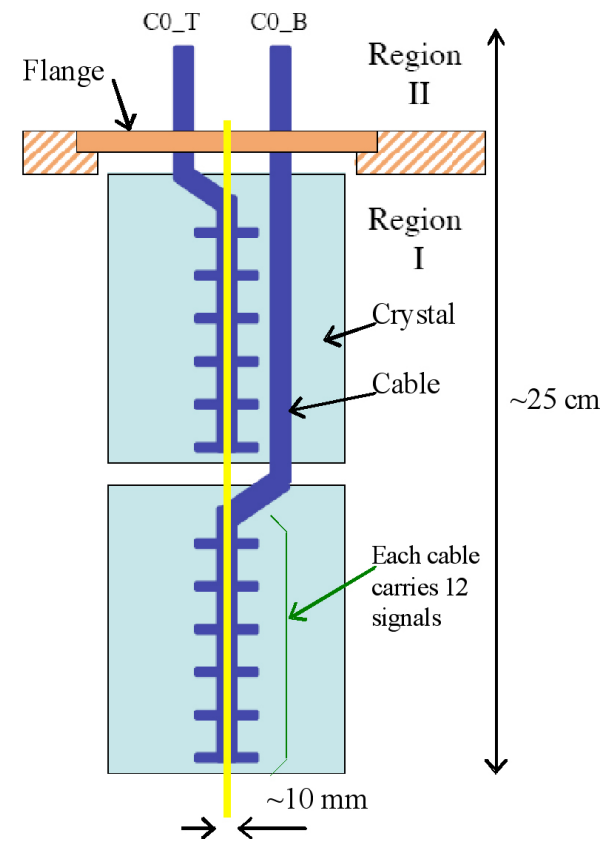

Figure 3: Sketch of the simplified crystal interconnect for this work.

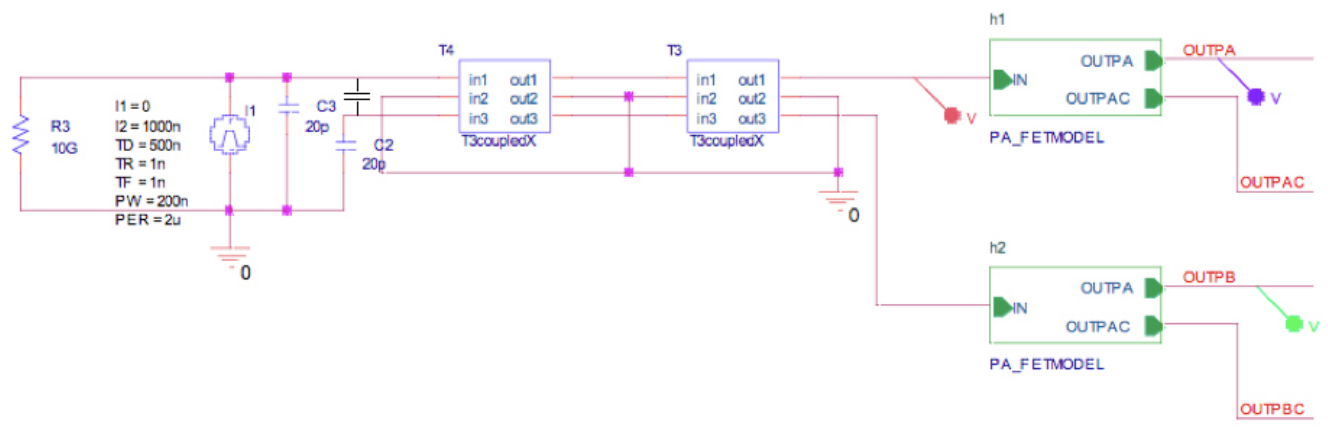

Figure 4: Sample simulation configuration.

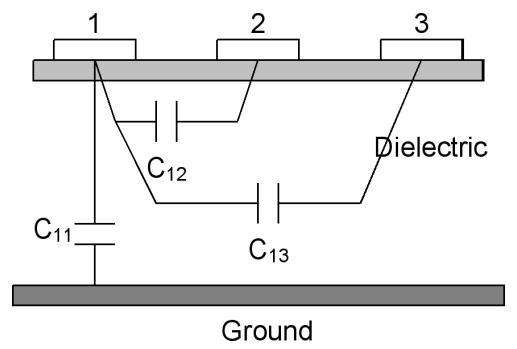

Figure 5: Traces and capacitances 
- Trace pitch: $400 \mu \mathrm{m}$

- Trace width: $50 \mu \mathrm{m}$

- Length: $\sim 25 \mathrm{~cm}$

- Distance from ground: $0.5 \mathrm{~mm}$

This set of dimensions generates the capacitance matrix as shown in Table 1, where $C_{i i}$ is the capacitance between the trace and the ground and $C_{i j}$ with $i \neq j$ is the capacitance between the traces (see Fig. 5). The traces are numbered from 1 to 3 , and trace number 2 is the trace in the middle.

Table 1: Short Cable Capacitance Matrix: $\mathrm{pF} / 25 \mathrm{~cm}$

\begin{tabular}{|c||c|c|c|}
\hline$C_{i j}$ & 1 & 2 & 3 \\
\hline \hline 1 & 4.5 & 1.5 & 0.3 \\
\hline 2 & 1.5 & 4.5 & 1.5 \\
\hline 3 & 0.3 & 1.5 & 4.5 \\
\hline
\end{tabular}

The dimensions of the long cable (wider than short cable) are the following:

- Trace thickness: $17 \mu \mathrm{m}$

- Trace pitch: $1100 \mu \mathrm{m}$

- Trace width: $100 \mu \mathrm{m}$

- Length: $100 \mathrm{~cm}$

- Distance to Ground: $1 \mathrm{~mm}$

Table 2 shows the capacitance matrix for the long cable. The conclusion from this study is that the long cable has larger contribution to crosstalk and capacitance to ground than the shorter cable, even if its traces are farther apart. We decided to concentrate our effort in studying and optimizing signal propagation along the long cable for the rest of this work, as what we learned from there should be applicable to the short cable as well.

Table 2: Long Cable Capacitance Matrix: pF/100cm

\begin{tabular}{|c||c|c|c|}
\hline$C_{i j}$ & 1 & 2 & 3 \\
\hline \hline 1 & 15.0 & 3.3 & 0.6 \\
\hline 2 & 3.3 & 15.0 & 3.3 \\
\hline 3 & 0.6 & 3.3 & 15.0 \\
\hline
\end{tabular}

\subsubsection{The Testbed Prototype Cable}

Based on simulation study, we have designed and manufactured (via a cable vender) a prototype cable to study properties of the long cable when connected to a charge sensitive preamplifier. This wide cable is actually made up of three independent long cables. Fig. 6 shows a sketch of the cable we had designed. It has three different regions, each region with different trace width and trace spacing (refer to Table 3). Each one of these regions will have 10 traces. Here are the characteristics of the cable:

- Length: 30"

- Trace: $17 \mu \mathrm{m}$ copper $\left(0.5\right.$ ounce/ $\left./ \mathrm{ft}^{2}\right)$ 


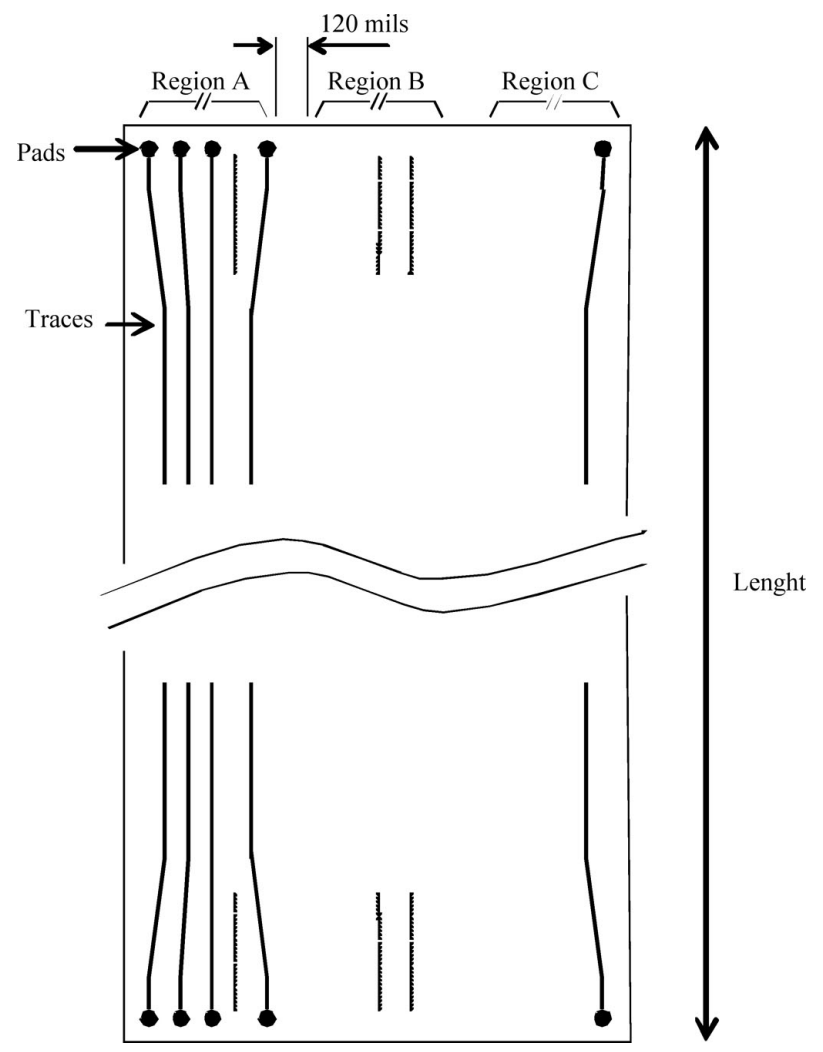

Figure 6: Trace pattern of the long prototype cable.

Table 3: Trace width and spacing of the long prototype cable

\begin{tabular}{|c|c|c|}
\hline Region & Trace width (mil) & Trace spacing (mil) \\
\hline \hline A & 4 & 40 \\
\hline B & 4 & 60 \\
\hline C & 6 & 40 \\
\hline
\end{tabular}

- Base: $25 \mu \mathrm{m}$ adhesiveless polyimide

- Cover layer: none

- Number of trace groups: equivalent to 3 independent long cables

This prototype cable, with a very thin substrate, was installed inside a shielded environment and used as the main testbed cable for this study.

\subsection{Measurements Without a Detector}

We have assembled a test setup to measure the noise performance and cable crosstalk of the present system. Fig. 7 shows a photo of the equipment. The cable and amplifiers (in this case, GRETINAtype amplifiers) are assembled inside a closed aluminum enclosure to shield them from other potential $\mathrm{RF}$ interferences. One side of the cable is connected to the amplifiers, while the other side is connected to BNC connectors where we can inject signals, as calibration charges. 


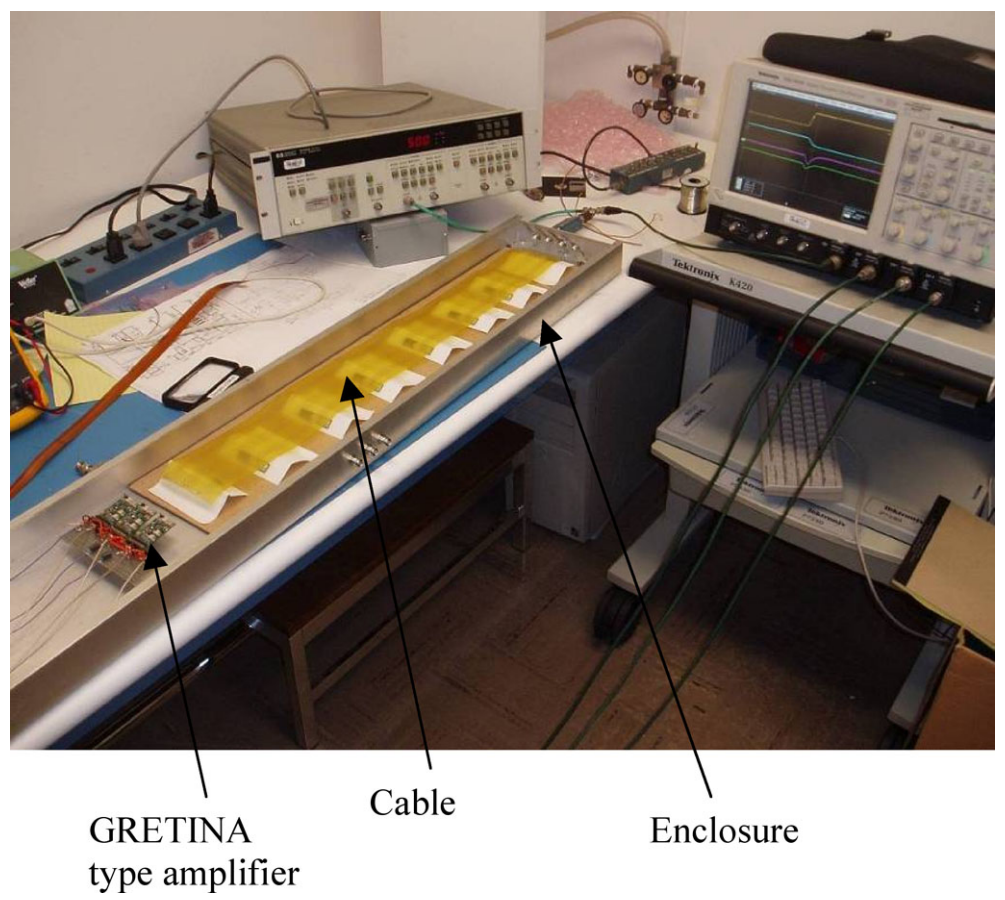

Figure 7: Bench test setup for the long cable measurements.

\subsubsection{Risetime Measurements}

When the test setup described above was powered up, the amplifiers started oscillating. This is due to the fast design speed of these amplifiers, which required further dumping. After fine tuning the damping characteristics, we measured the following rise-times with the cable connected to a capacitive load to simulate the detector capacitance:

- Load $\approx 2 \mathrm{pF} \rightarrow t_{R}=50 \mathrm{~ns}$

- $\operatorname{Load} \approx 30 \mathrm{pF} \rightarrow t_{R}=130 \mathrm{~ns}$

For this amplifier type the literature shows that the rise time is given by [10]:

$$
t_{R(10-90 \%)}=\frac{2.2}{g_{m}} \frac{C_{V}}{C_{F}}\left(C_{D}+C_{g s}\right),
$$

where:

- CV : Compensating capacitance

- $\mathrm{CF}$ : Feedback capacitance

- CD : Detector capacitance

- $\mathrm{C}_{g s}$ : FET gate-source stray capacitance

- $\mathrm{g}_{m}$ : FET transconductance

One expects a slower risetime will not affect the very robust segment hit multiplicity information, but the accuracy in position reconstruction could be affected. One way to increase the rise time of this class of amplifier is to increase the open loop gain. In the tested configuration, the gain is given by the FET transconductance $g_{m}$. Such an option is presented in Reference [9] for a MAFYA-type preamplifier design. 


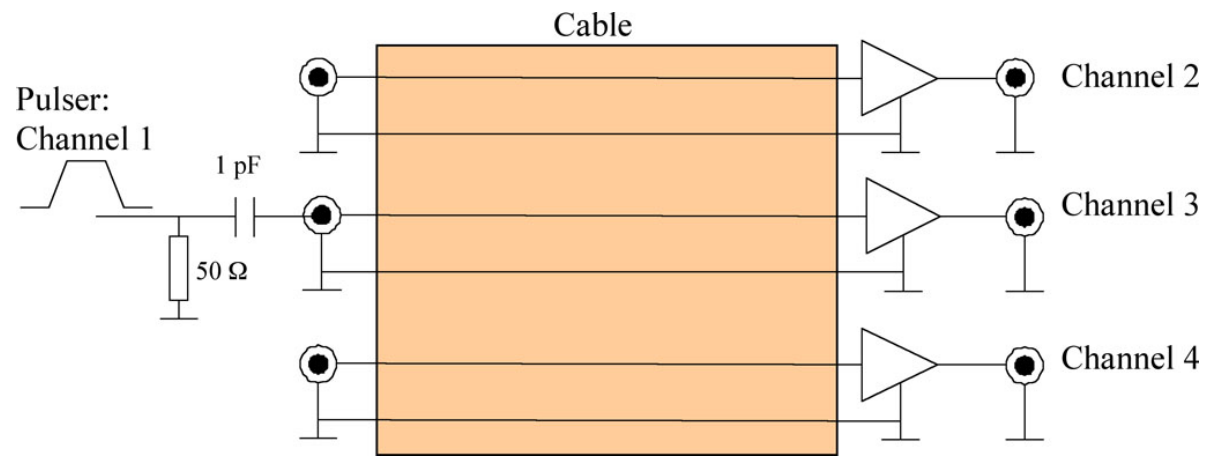

Figure 8: Test cable connection for crosstalk measurement.

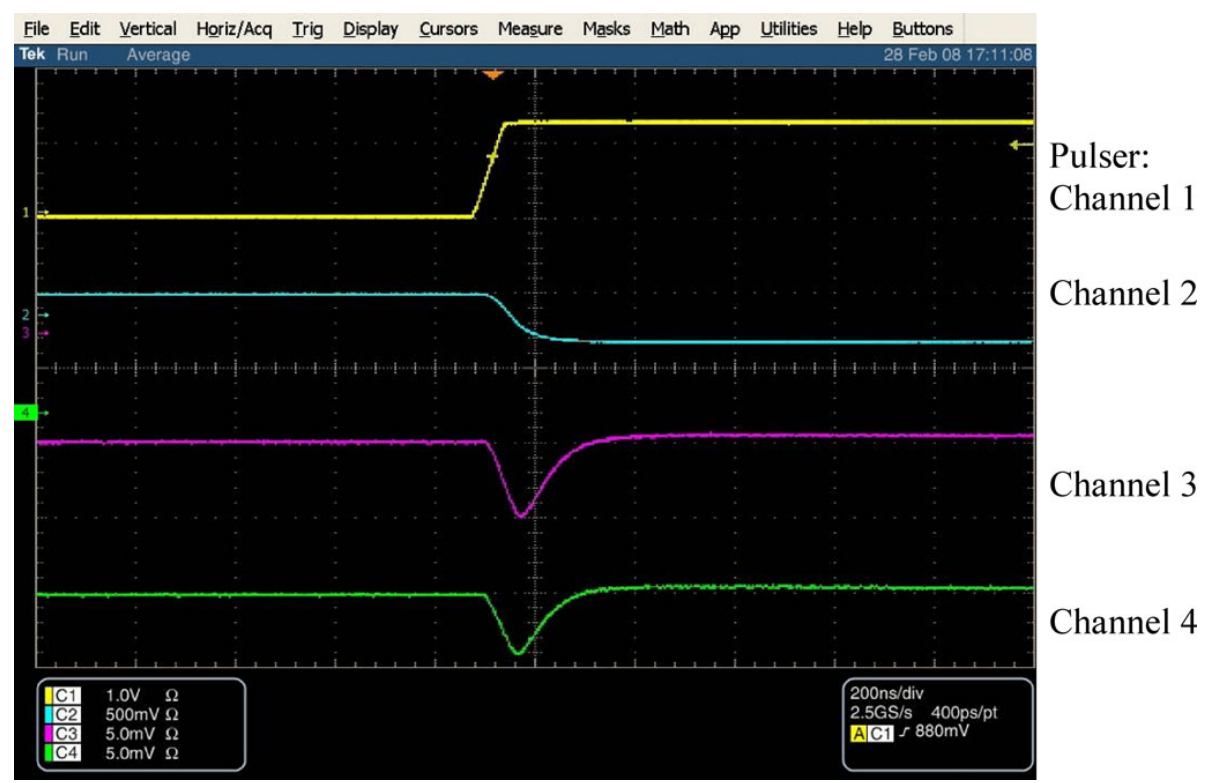

Figure 9: Scope traces of sample signal crosstalk.

\subsubsection{Crosstalk Measurements}

Fig. 8 shows a schematic of the interconnections we used to measure crosstalk on the cable. A signal is injected on the trace adjacent to two other signal traces that have no signal injected. This figure also shows the oscilloscope channels we use for this measurement.

We have measured two types of crosstalk, one known as differential crosstalk (caused by the fast transition of the charge on the cable) and the other known as integral crosstalk [10]. In these tests we used the traces on the A and B regions of the cable (see Table 3). The waveforms of the differential crosstalk for region B are shown in Figure 9. The risetime of the pulser is $30 \mathrm{~ns}$, and the voltage setup of the oscilloscope is $500 \mathrm{mV} /$ div for channel B and $5 \mathrm{mV} / \operatorname{div}$ for channels $\mathrm{A}$ and $\mathrm{C}$. The differential crosstalk is highly dependent on the rise time of the charge injection pulser and the frequency response of the preamplifier.

Table 4 shows the measurement for differential and integral crosstalk for regions A and B of the cable (see Table 31). One can observe that with more spacing the differential crosstalk decreased and, within the precision of our instrumentation, the integral crosstalk between these two zones did not change. More precise instrumentation would likely show that traces with more spacing would 
Table 4: Differential and integral crosstalk of the long prototype cable

\begin{tabular}{|c|c|c|}
\hline Trace Spacing $(\mathrm{mm})$ & Differential (percent) & Integral (percent) \\
\hline \hline 1.0 & 2.7 & 0.2 \\
\hline 1.5 & 1.5 & 0.2 \\
\hline
\end{tabular}

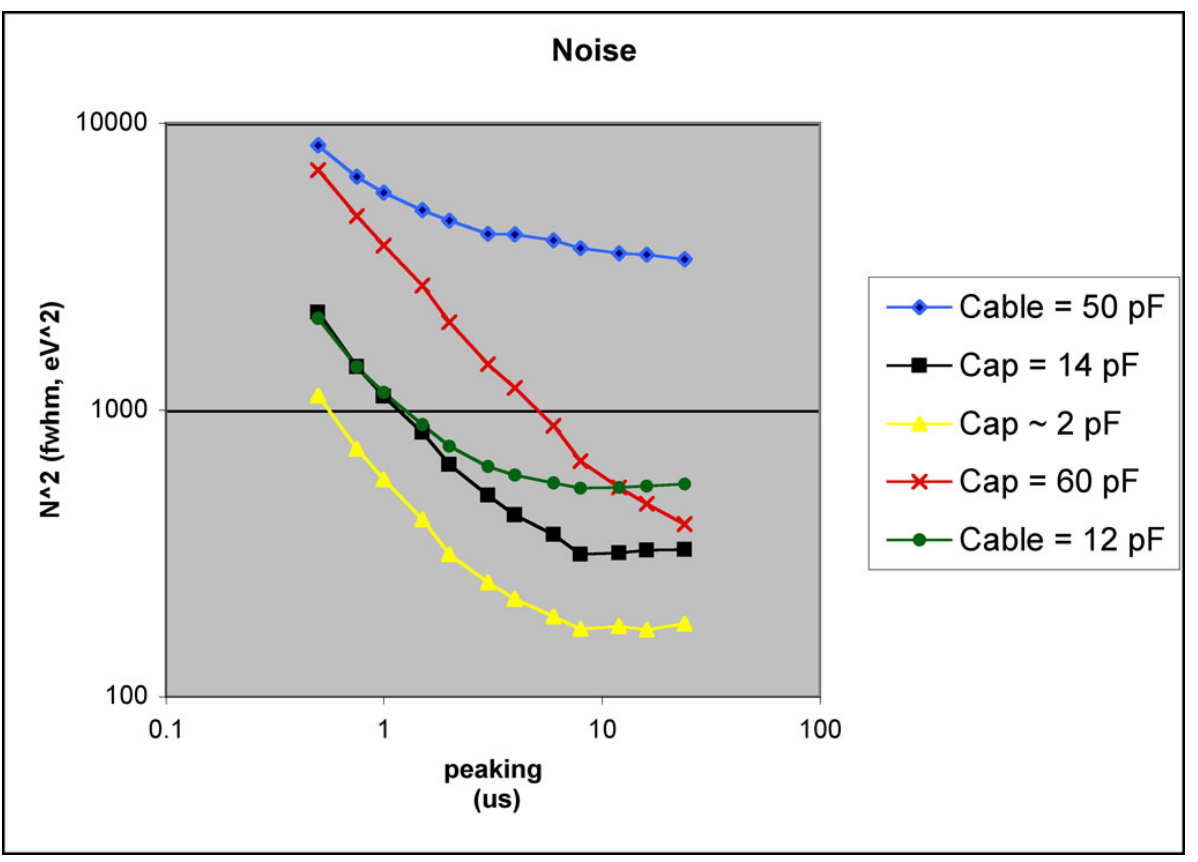

Figure 10: Bench top noise measurements for two different cables and three different input capacitors when connected to a GRETINA-type amplifier.

have smaller integral crosstalk.

\subsubsection{Noise Measurements}

We have also measured and compared the electronic noise performances for two Kapton-based multitrace flex cables and several input capacitors (simulating the detector load) when connected to a GRETINA-type amplifier. One of the Kapton cables is the one specified here, and we tested region B. The other is a cable with similar length, with $150 \mathrm{um}$ dielectric and coverlay thickness and $50 \mathrm{pF}$ capacitance between traces. Fig. 10 shows the noise performance of the cables and capacitors as a function of shaper peaking time.

One can observe that the noise performance of the preamplifier is very dependent on the input capacitance - larger input capacitances increases the amplifier noise. Also, observe that the noise performance of the $50 \mathrm{pF}$ Kapton cable is worse than a similar $60 \mathrm{pF}$ capacitor. This can be explained by the losses associated with Kapton. The loss tangent (a measure of the dielectric loss) of Kapton is typically 0.002 , compared with CuFlon that is typically 0.0001 (20 times smaller). Also, observe that a similar effect is also visible for the $12 \mathrm{pF}$ cable that we designed when compared with a 14 $\mathrm{pF}$ capacitor. However, the degradation in performance is not so pronounced as for the $50 \mathrm{pF}$ cable, because the amount of Kapton is substantially less ( $\mu \mathrm{m}$ compared with $20 \mu \mathrm{m}$ ).

It is important to note too that CuFlon, a polymer based on Teflon, should have the added 

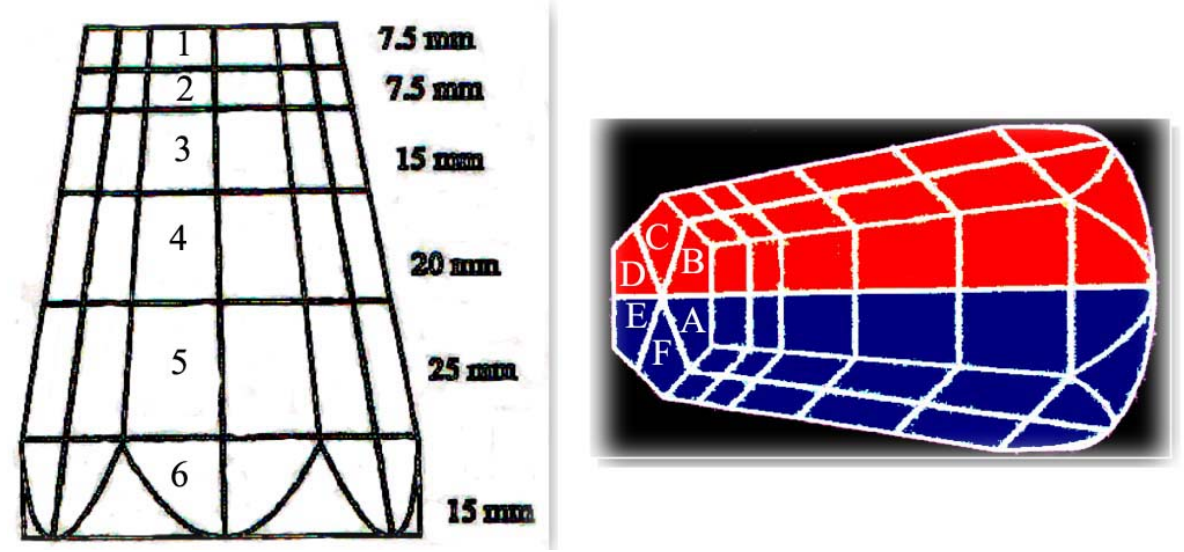

Figure 11: The 6x6 Segments of the tapered GP2 prototype detector. Half of the segments are configured with warm FETs (red color).

advantage of lower background radiation. Another possible candidate cable substrate material is polyethylene naphthalate (PEN).

\subsection{Measurements with a Segmented Ge Detector}

In January 2008 a N-type segmented coaxial detector, GP2, was loaned to the MAJORANA collaboration at LBNL from the GRETINA collaboration. GP2 (GRETINA Prototype II) was a testbed detector for the GRETINA Group at LBNL for developing and optimizing the final detector module. It has gone through a few crystal reprocessing cycles by the manufacturer with different cold and warm FET electronics implementation.

This detector is divided into thirty-six segments, as shown in Fig. 11. The signals originating from these segments are further divided into two categories. Half of the segments are fed through warm FETs located outside the cryostat as shown in Fig. 12, and the other half are fed to cold FETs located within the cryostat. The original purpose of this separation was to find the optimal placement of the FET and to quantify the impact on the signal resolution. The central contact signal from the entire crystal is also read out with two internally-paired channels. These channels are known as the full volume (FV) channels and are both warm. The nomenclature of the segments is defined as "LN", with $\mathrm{L}=(\mathrm{A}, \mathrm{B}, \mathrm{C}, \mathrm{D}, \mathrm{E}$ or $\mathrm{F})$ and $\mathrm{N}=(1,2,3,4,5$ or 6$))$ in Fig. 11. Segments wtih $\mathrm{L}=\mathrm{B}, \mathrm{C}$, and $\mathrm{D}$ are instrumented with warm FETs, while segments with $\mathrm{L}=\mathrm{E}$, F, and $\mathrm{A}$ are instrumented with cold FETs. This detector had stayed at room temperature without being operated for approximately one year. It was transported from its storage place to Bldg. 70A and then cooled down for the present signal measurement task.

Once the detector was cooled, issues were encountered that affected the stability of the signal. One of these issues was the random occurrence of pulse with very large amplitude that resembled breakdown (Fig. 13). These pulses are of high frequency and indicate some possible problems with the crystal. The detector was pumped down to obtain the best possible vacuum, but these breakdowns persisted. The detector was finally baked, a process that involves bringing the detector to a temperature high enough ( 80 degree celsius) to get rid of some debris on the surface of the crystal while at the same time vacuum pumping. The setup for this detector conditioning process is pictured in Fig 14. This baking process solved the breakdown issue. One of the warm segment had a leakage current value out of norm before the baking was considered at fault. After baking, this segment leakage current value was within norms.

Another issue that affects the stability of the detector is microphonic vibrations from the sur- 


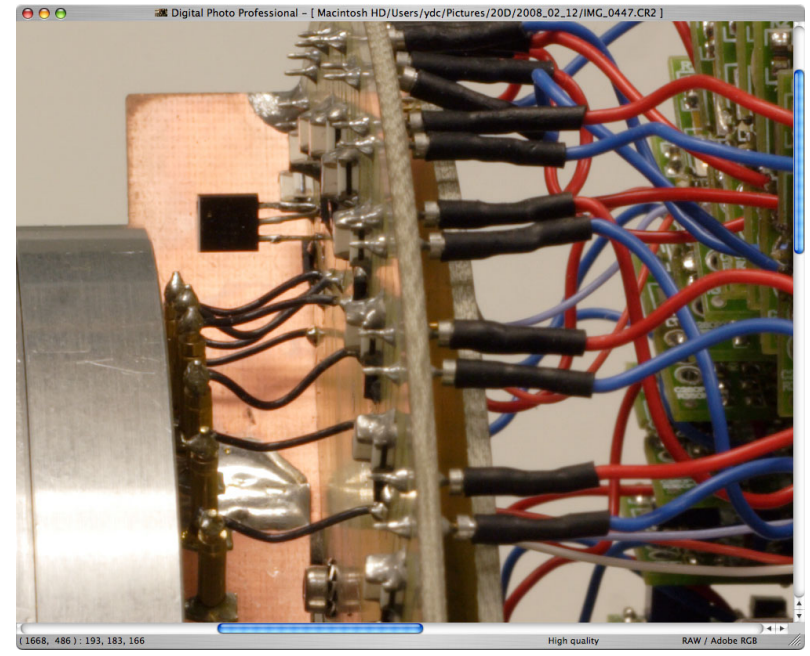

Figure 12: The warm FET section of GP2

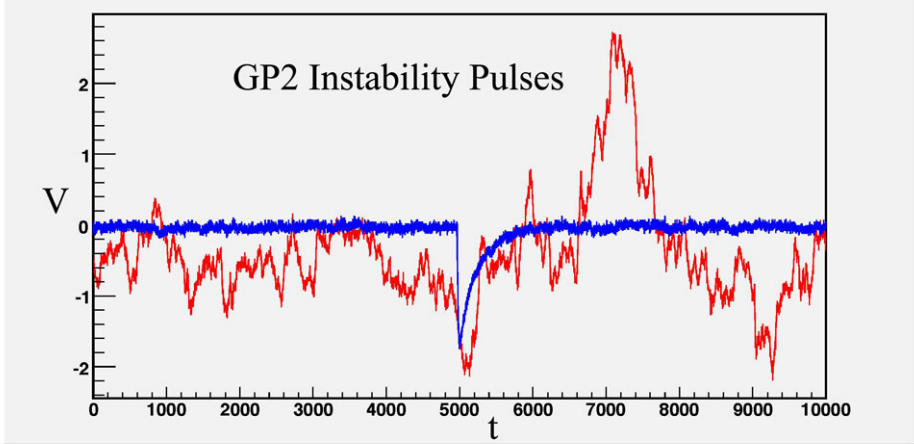

Figure 13: Example scope tracess (blue: segment, red: FV) showing the instability behavior of the GP2 detector. 


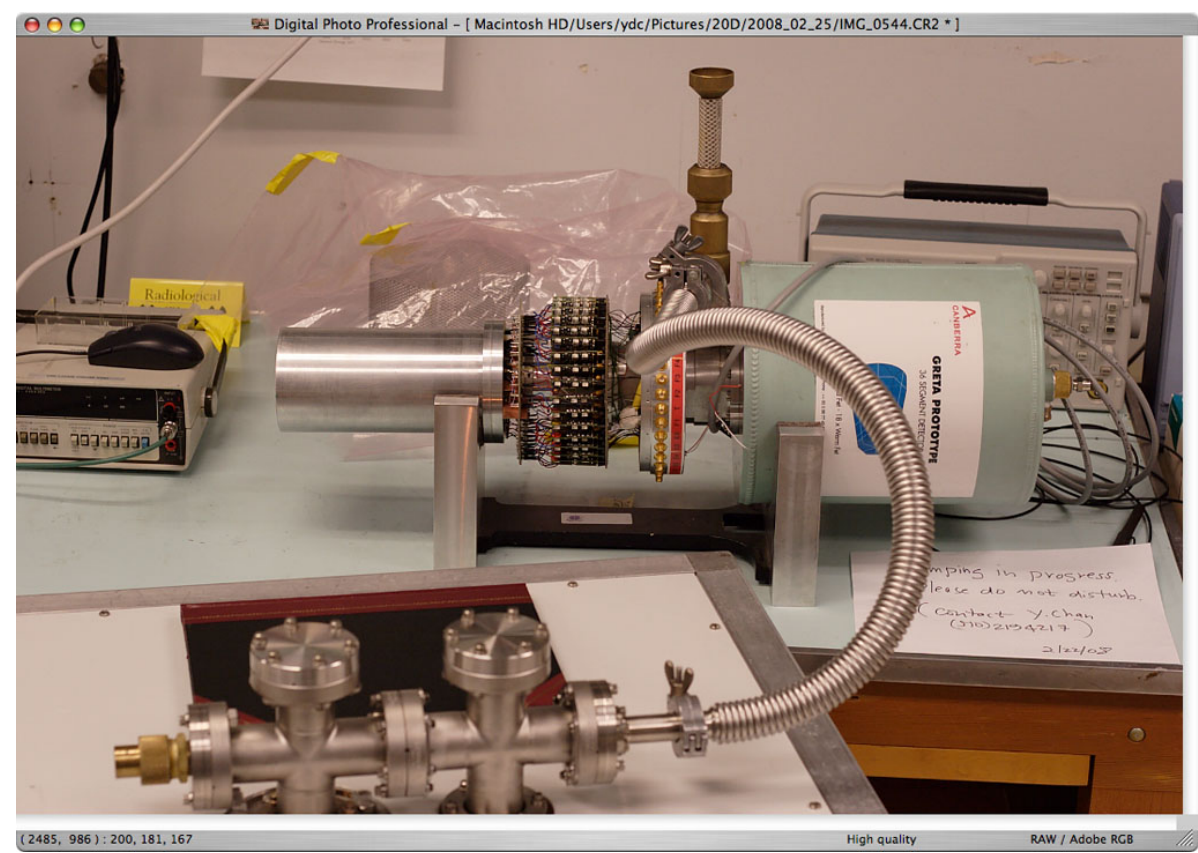

Figure 14: Attempt to re-condition the GP2 detector to improve its stability.

rounding environment. While they do not pose a danger to the crystal, they have a direct impact on the resolution of the detector. The high-density and temporary nature of the GP2 electronics make them particularly sensitive to this issue (see Figs. 12 and 15 . The electronics can act as an antenna and can pick up electromagnetic noise from the surrounding instruments. Mechanical vibrations due to things like air currents and human motion can also generate microphonics. This issue can be fixed without ramping down the detector and adjusting the electronics configuration. More permanent solutions are currently being worked on. After fixing a few minor, obvious microphonics problems, the detector segments were tested for stability. It was found that the detector was stable for periods of time long enough to proceed with the cable studies.

The warm segments C4 and D4, which were among the segments with more stable behavior, were selected for the following signal comparison study. The FET to preamp connections for C4 and D4 were modified and interfaced to the prototype cable. We connected the segment to the long cable and amplifier described in the previous section. Fig. 16 shows this assembly. We opened a small penetration on the detector cover and routed two wires with the signals for segments C4 and D4 to the amplifier and cable enclosure. We then connected these wires to the cable and we observed the output of the amplifiers on the other side of the enclosure. We used region B of the cable for these tests.

\subsubsection{Energy Resolution}

We have measured and compared the energy resolution of the $\mathrm{C} 4$ segment for various scenarios. We used a ${ }^{60} \mathrm{Co}$ source, tuned the shaper amplifier to $6 \mu$ s peaking time, and observed the resolution of the $1173 \mathrm{keV}$ line. Fig. 17 and Fig. 18 , show the ${ }^{60} \mathrm{Co}$ source energy spectra taken by the $\mathrm{C} 4$ segment with its original connection and with the long prototype cable connected, respectively. Fig. 19 shows the same spectrum for the central contact, which was connected to a warm FET. The FV resolution of the GP2 detector was not very good due to the many issues discussed above.

We also measured the noise of the amplifier inside the enclosure with the 30 " cable, but without 


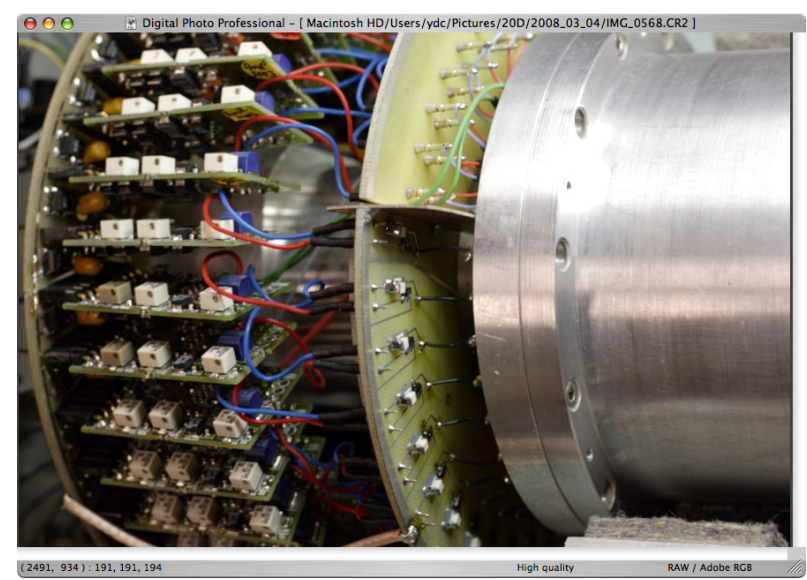

Figure 15: The preamplifier section of GP2.

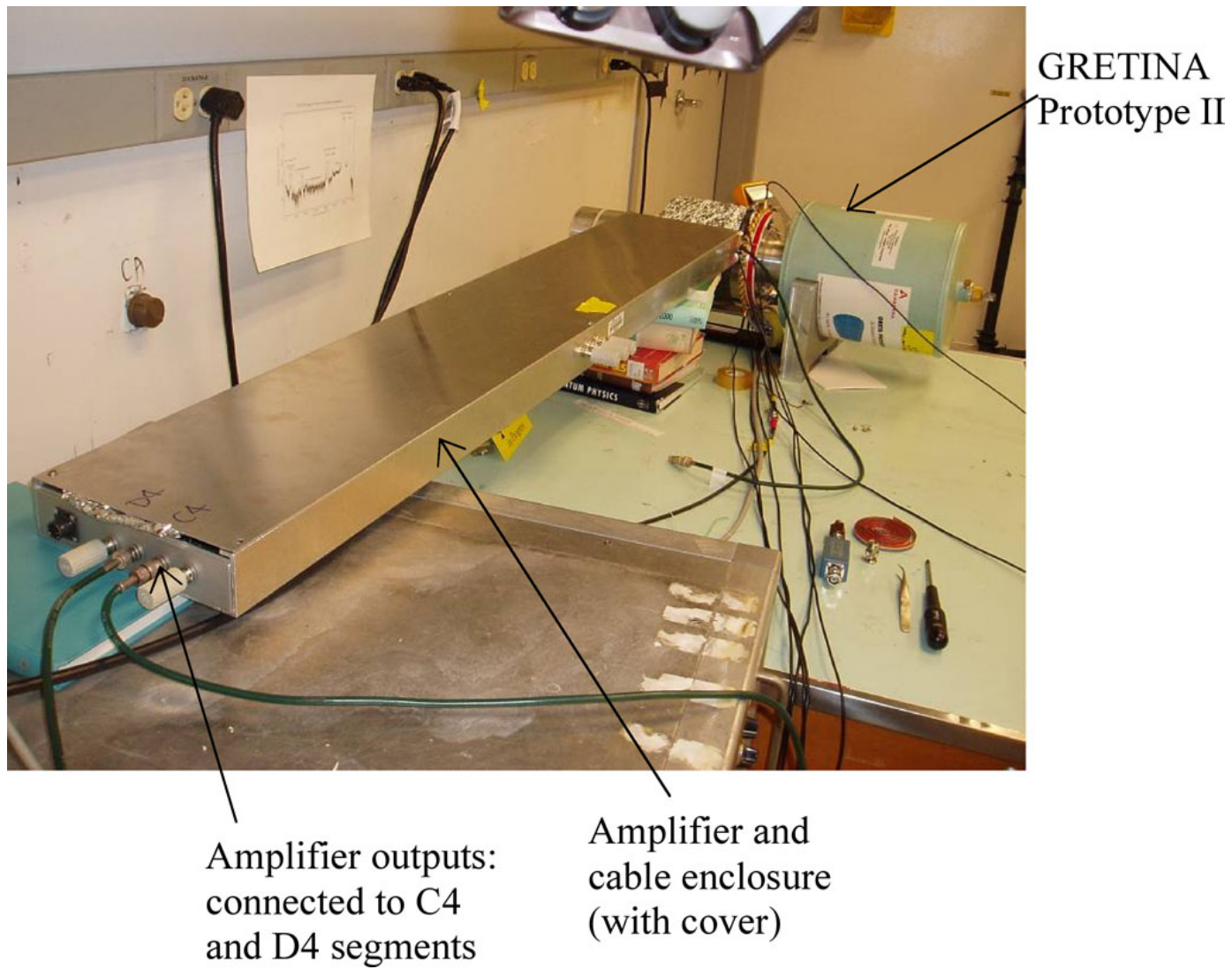

Figure 16: Connecting the prototype cable to the GP2 detector. 


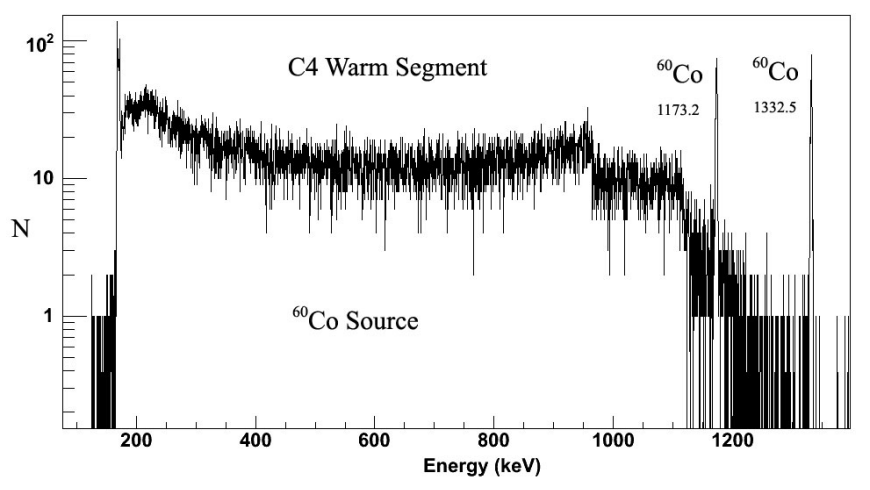

Figure 17: Energy spectrum for ${ }^{60} \mathrm{Co}$ from segment $\mathrm{C} 4$ with a warm FET (unmodified).

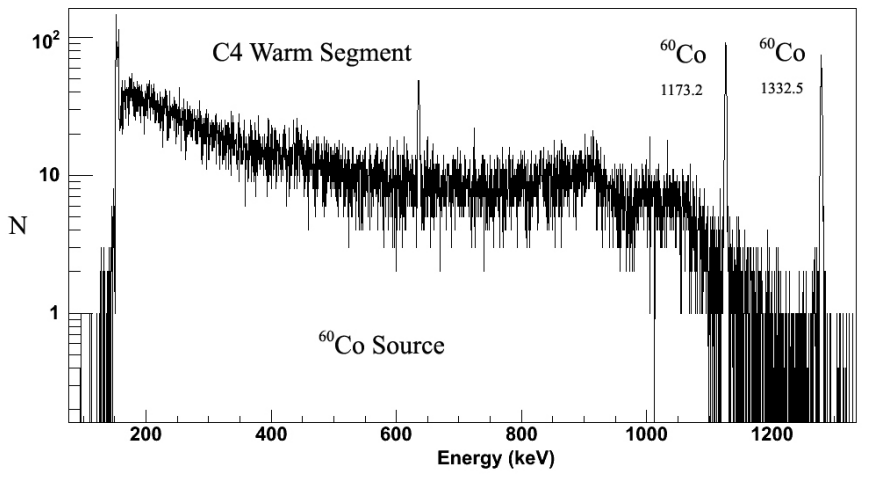

Figure 18: Energy spectrum for ${ }^{60}$ Co from segment $\mathrm{C} 4$ with a warm FET and connected to the long prototype cable. The structure around channel 630 was due to another gamma source in the proximity of the GP2 detector during data taking time.

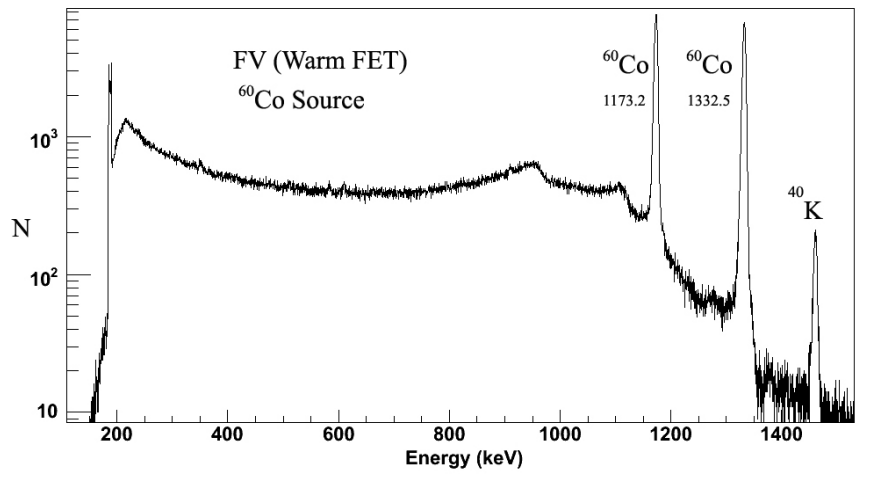

Figure 19: Energy spectrum for ${ }^{60} \mathrm{Co}$ from the central contact (FV, full volume) of the GP2 detector. The FV is configured with a warm FET. 
Table 5: Energy resolution comparison

\begin{tabular}{|c|c|}
\hline Preamp location & $\begin{array}{c}\text { Energy Resolution (FWHM, keV) } \\
{ }^{60} \mathrm{Co}, 1173 \mathrm{keV} \text { line }\end{array}$ \\
\hline \hline Original position inside detector module & 2.5 \\
\hline Inside enclosure but without the 30" cable & 3.0 \\
\hline Inside enclosure, interfaced segment through the 30" cable & 3.4 \\
\hline
\end{tabular}

the detector connected. This setup is the same as the one used to characterize the amplifier noise as reported in Section 3.2, with one difference - we had changed to a noisier room with more equipment running. In this situation the noise increased to $1.5 \mathrm{keV}$, again with $6 \mu$ s peaking time. Observe that this performance is substantially worse than that reported in Section 3.2 , where the noise was $\sim 0.8 \mathrm{keV}$.

The results of the measurements, listed in Table 5 indicated that the performance degraded substantially from the original amplifier position (inside the GP2 detector) as compared to the new installation with the preamplifier inside the enclosure and connected to the detector via a 30" cable (from $2.5 \mathrm{keV}$ to $3.4 \mathrm{keV}$ ). This can not be explained by the noise added by the cable $(0.8 \mathrm{keV})$. These tests suggest that the mechanics of the detector assembly will be critical to damp microphonics and to provide a RF-free environment inside the cryostat.

\subsubsection{Crosstalk}

We have compared the crosstalk of channels $\mathrm{C} 4, \mathrm{D} 4$ and $\mathrm{E} 4$, which are adjacent channels in the $z$ direction of the crystal. Channels C4 and D4 are instrumented by the amplifier inside the enclosure and connected to the 30" cable. Channel E4 uses an amplifier inside the detector enclosure. We can inject charge into D4 using a pulser and capacitor, and observe the crosstalk in the adjacent channels $\mathrm{C} 4$ and $\mathrm{E} 4$. The pulse generator was set with $t_{r}=25 \mathrm{~ns}$.

Fig. 20 shows an example of the differential crosstalk we measured. This one is between D4 (generating the crosstalk) and C4 (sensitive to the signal in D4). We can now compare the crosstalk without the cable and the crosstalk with the cable. For this scenario, the crosstalk degraded from $\sim 4 \%$ to $\sim 5 \%$. Observe that this measurement is compatible with the differential crosstalk measured in Section 3.3.2, where the differential crosstalk for the cable region A was 1.5\%: $5 \%$ is approximately the sum of the crosstalk without the cable plus the previous measurement.

It is interesting to report about another test result that shows how sensitive this system is to crosstalk caused by very small capacitances. Fig. 21 shows the differential crosstalk we measured when we first started these studies. Observe that an integral crosstalk is injected in $\mathrm{C} 4$ (the tail of the waveform) with amplitude $\sim 1 / 3$ of the differential crosstalk, which we know is not likely. To investigate the origin of this integral crosstalk we modeled in SPICE the behavior of the detector/amplifier connections (see Fig. 22). We are injecting charge into the preamplifier A and detecting charge into preamplifier $\mathrm{B}$. The $45 \mathrm{pF}$ and $10 \mathrm{pF}$ capacitors simulate the detector capacitance, and the PA_FETMODEL is the model of the preamplifier. But in this configuration we add a $20 \mathrm{fF}$ capacitor between the pulser and the input of preamplifier B. Figure 23 shows the result of this simulation, which gives a similar behavior as is found in Fig. 22. Eventually we were able to shield the pulser from the sensitive channel (C4) and obtain the plot reported previously. But note that very small capacitances will have substantial impact on crosstalk.

\subsection{SEGA Electronics Support}

The warm-FET scheme employed in the GP2 detector were deemed sufficient for the requirements of the SEGA detector. An 8-channel, warm-FET electronics sub-system (including preamps, HV 


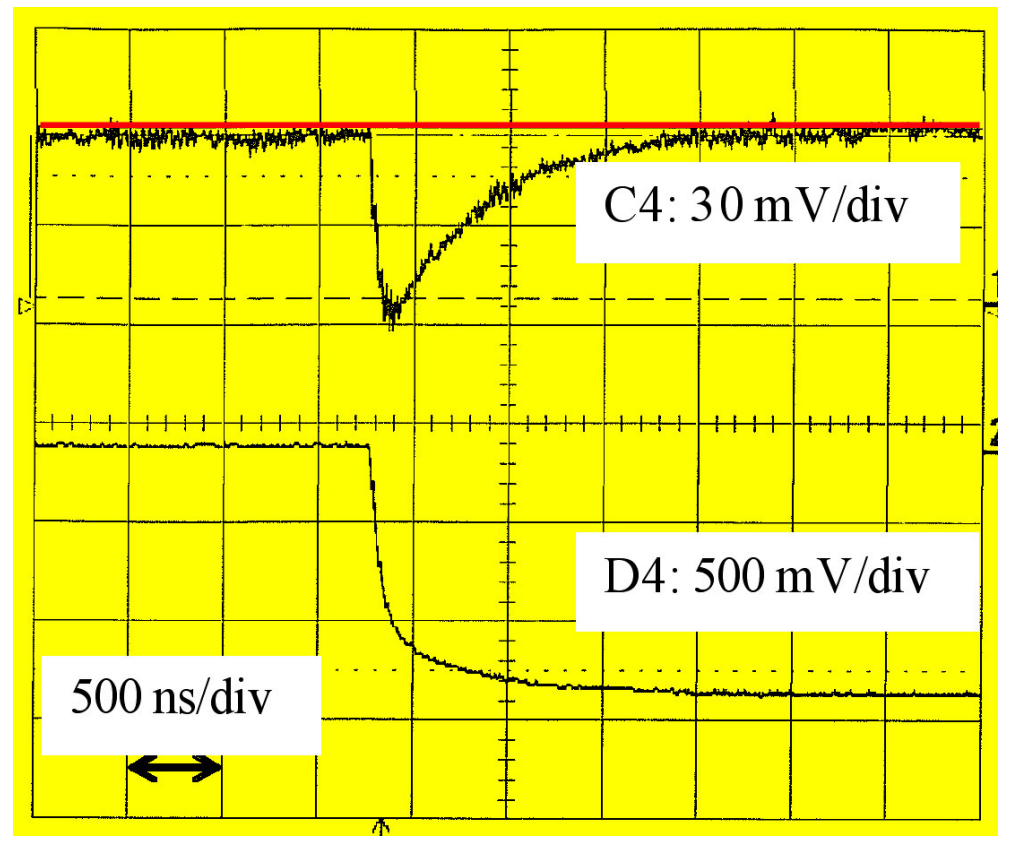

Figure 20: Differential crosstalk measurement. Channel C4: crosstalk; Channel D4: charge.

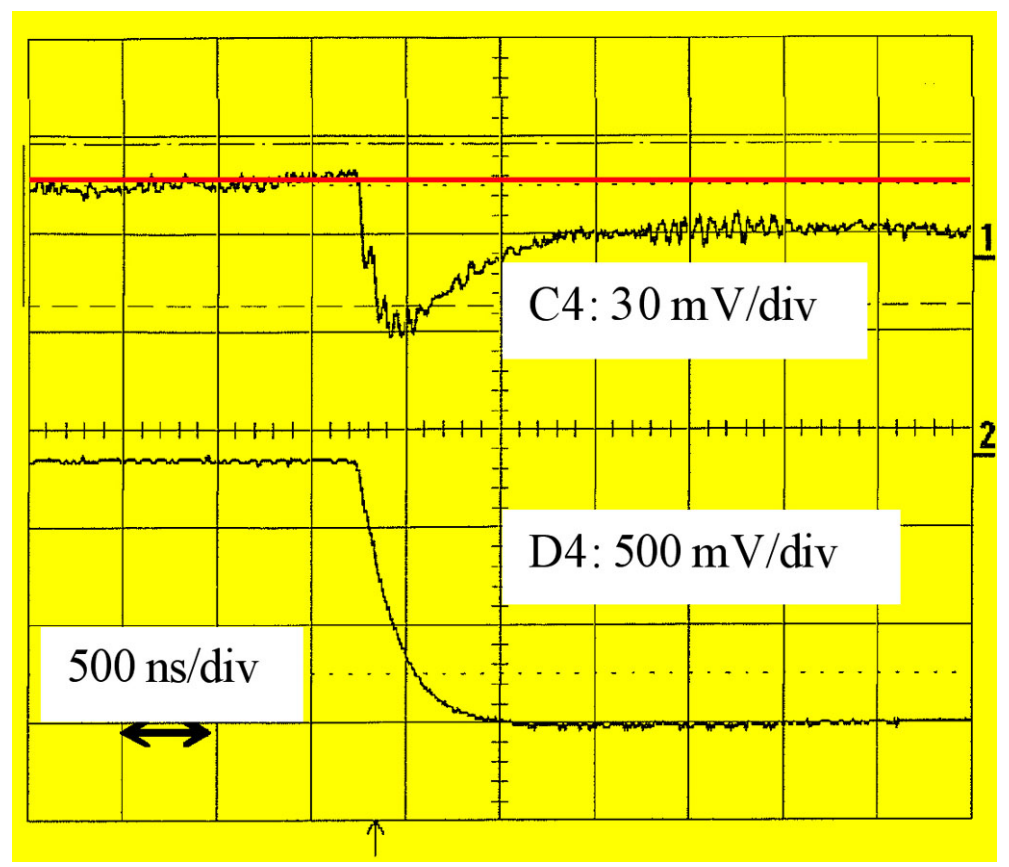

Figure 21: Differential crosstalk measurement with anomalously high integral cross talk. Channel C4: crosstalk; Channel D4: charge. 


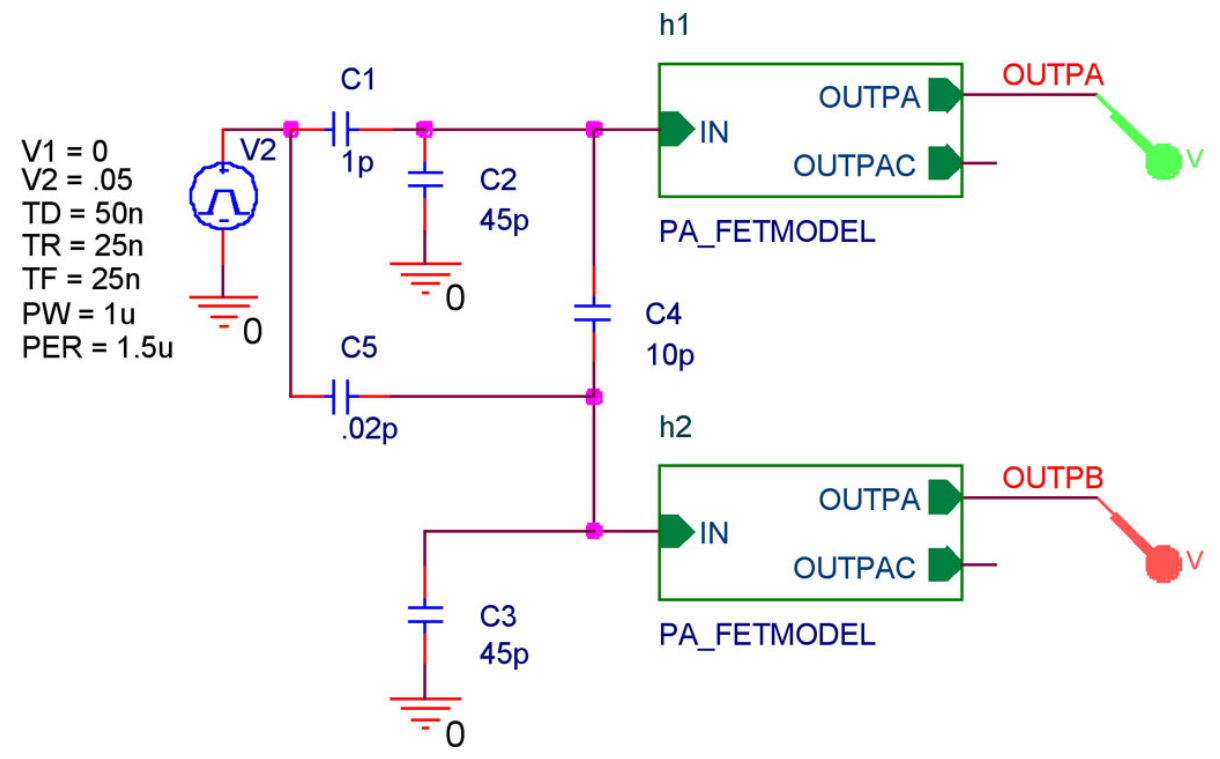

Figure 22: SPICE Model for the high integral crosstalk simulation.

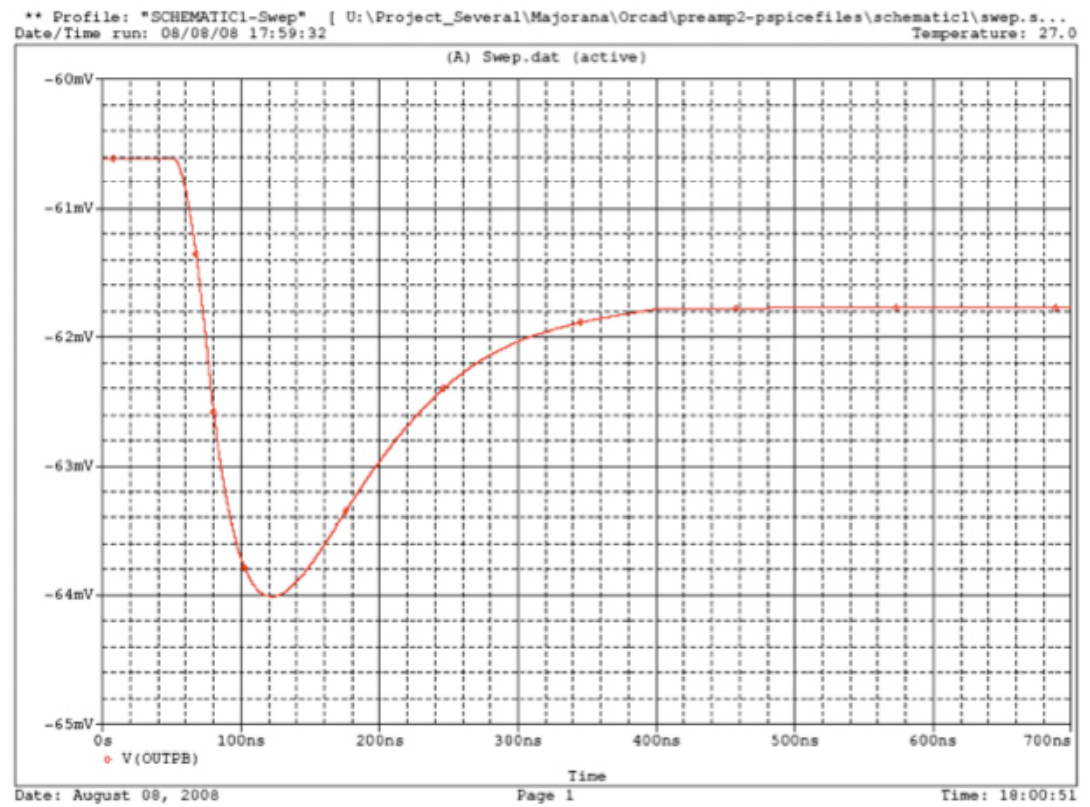

Figure 23: High integral crosstalk simulation. 


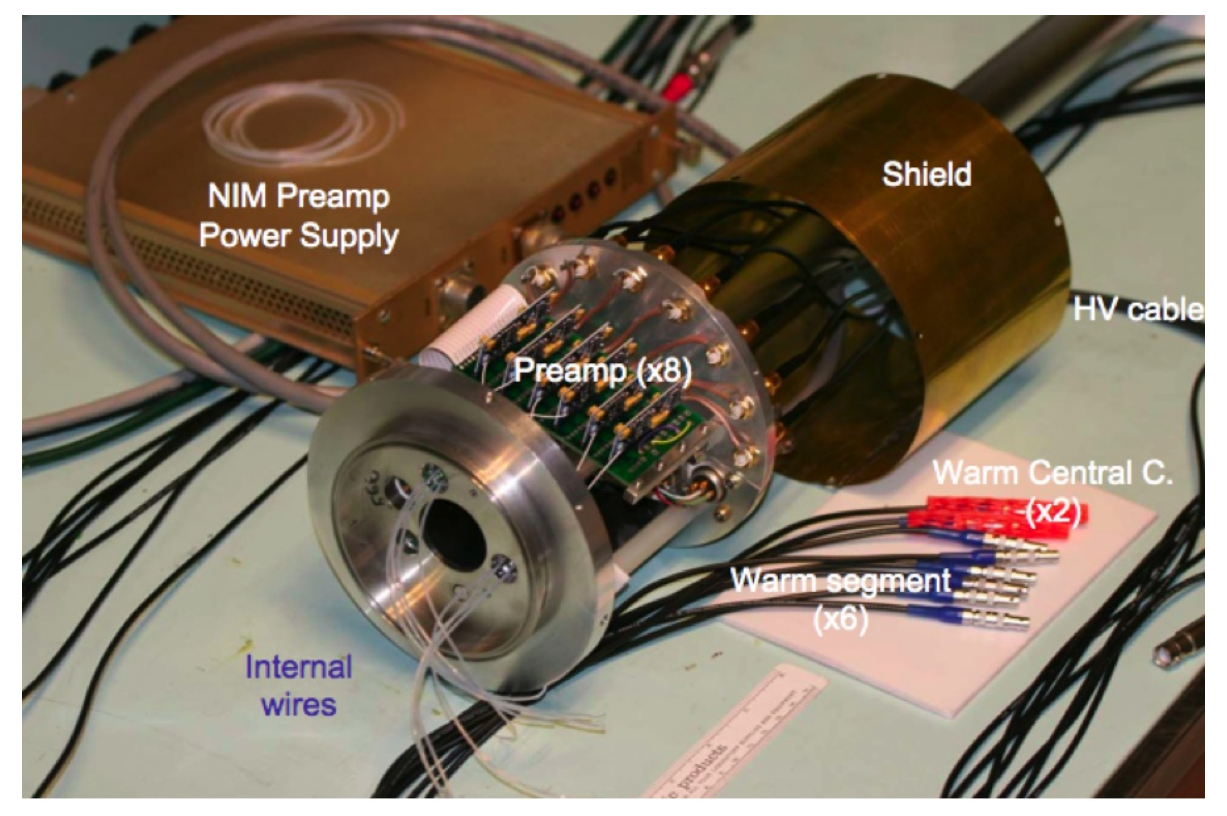

Figure 24: A picture of the SEGA test cryostat electronics.

filtering circuit, as well as external power supply) has been assembled for the SEGA transport cryostat. A picture of the setup is shown in Figure 24. The electronics have been tested and are ready for the SEGA detector task. 


\section{Alternative Capacitive Coupling for the Central Contact Readeout}

Capacitive coupling of the central contact is typically done as depicted in Fig. 25(a), where the high voltage capacitor $C_{C 1}$ decouples the detector bias voltage. This traditional method of decoupling requires reasonably large value capacitance to decrease the voltage drop over the capacitor. Typical values are around $5 \mathrm{nF}$, which results in a physically large component, implying more material and therefore potentially higher backgrounds.

There is an alternative method that has been employed for some time in which the decoupling capacitor is moved inside the feedback loop. This method is depicted in Fig. 25(b). The only requirement associated with this method is that $C_{C 2} \gg C_{F}$ [1] (this reference shows a circuit with a relationship of $C_{C 2}=20 \times C_{F}$ ). Since $C_{F}$ for this application is typically small (say $1 \mathrm{pF}$ ), $C_{C 2}$ can also have a small capacitance value, for example $\sim 20 \mathrm{pF}$. The advantage of this approach is that $C_{C 2}$ can be made physically smaller than $C_{C 1}$. However, now both $C_{F}$ and $C_{C 2}$ are high voltage capacitors, which requires a more massive $C_{F}$. Nevertheless, the total mass is still reduced as compared to the traditional capacitive coupling method.

We tested this approach by assembling the circuit in Fig. 25(b) and injecting charge into the front-end. We used components with the following values:

- $C_{F}=1 \mathrm{pF}$

- $R_{F}=1 \mathrm{G} \Omega$

- $C_{C 2}=5 \mathrm{pF}$ and $50 \mathrm{pF}$

We observed that with $C_{C 2}=5 \mathrm{pF}$ (see Fig. 26) the output voltage dropped by $\sim 4 \%$, indicating that $C_{C 2}$ is not fully coupling the signal in the amplifier input. When we increased $C_{C 2}$ to $50 \mathrm{pF}$ (see Fig. 27) we could not measure a voltage drop on the output of the amplifier.

Based on these promising simulation studies, a prototype AC-coupled front-end card based on this topology was designed and fabricated. Another bench top measurement was performed (see Fig. 28) to guide the layout design. The performance of the board was measured for a range of capacitance and resistance values. The prototype AC-coupled low mass front end board was fabricated with amorphus germanium resistors. Fig. 29 shows an illustration of the layout of the board, and Fig. reffig:lmfe1 shows the actual prototype fabricated locally at the LBNL detector laboratory. The final achieved resistance is $\sim 0.2 \mathrm{G} \Omega$ at room temperature. Fig. 31 demonstrates that the noise performance of this board is very good at room temperature.

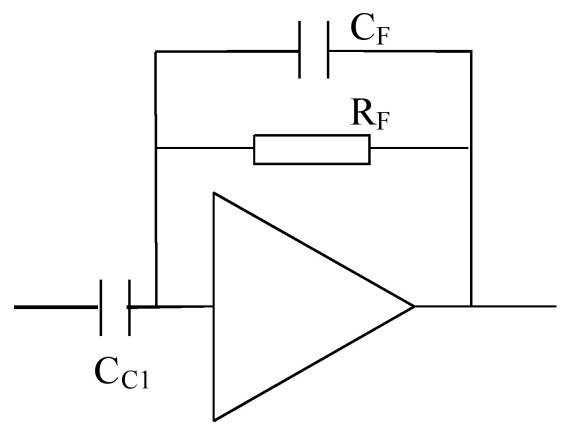

(a)

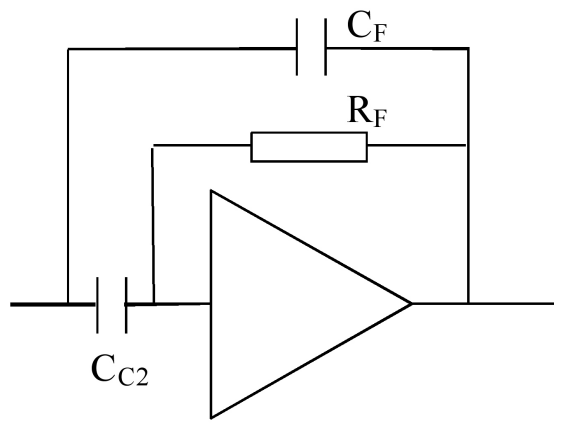

(b)

Figure 25: Detector AC-coupling configuration: (a) conventional option (b) small decoupling capacitance option. 


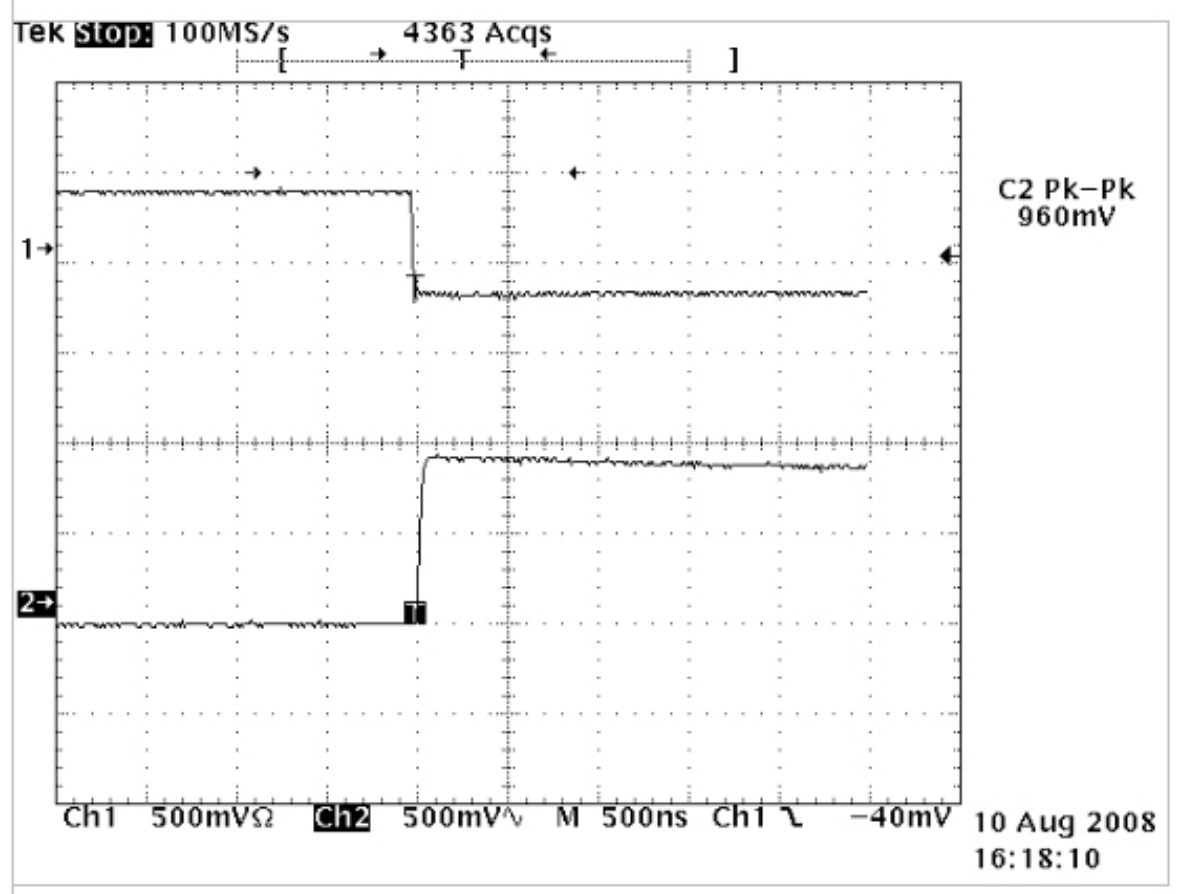

Figure 26: AC couple simulation for $\mathrm{C}_{C 2}=5 \mathrm{pF}$. Note the $\sim 4 \%$ voltage drop.

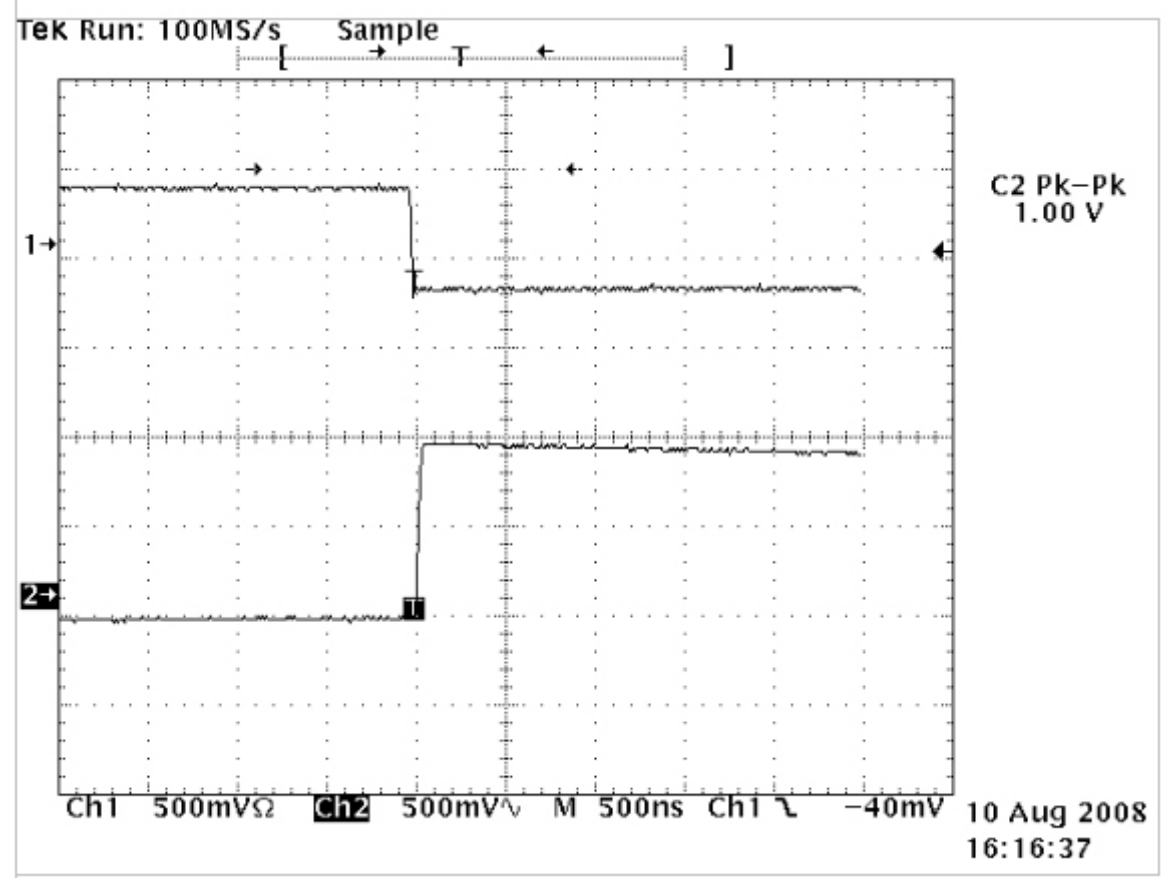

Figure 27: $\mathrm{AC}$ couple simulation for $\mathrm{C}_{C 2}=50 \mathrm{pF}$. No voltage drop observed. 

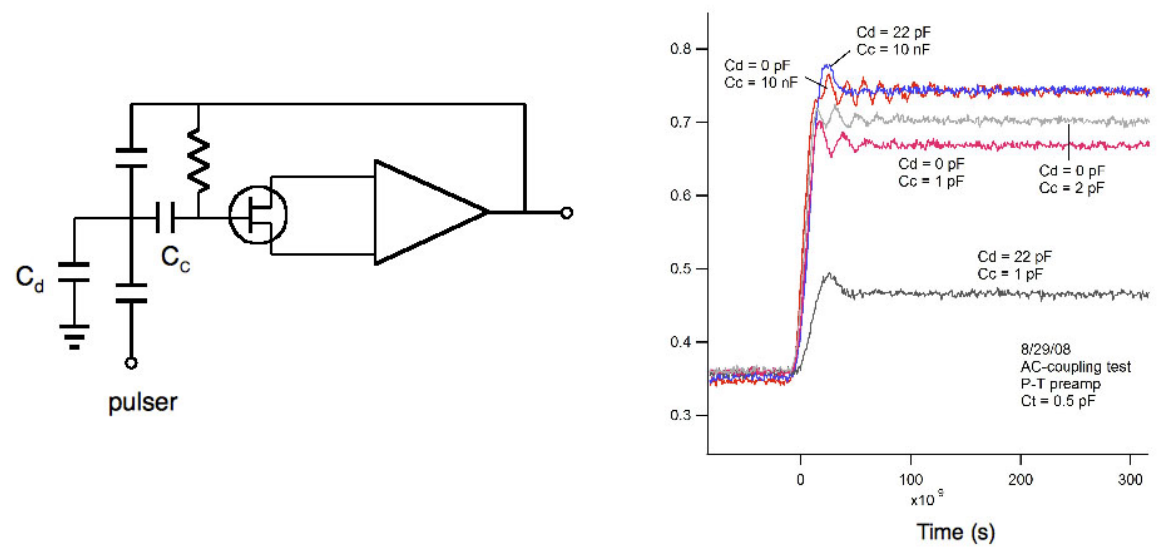

Figure 28: Another pre-fabrication bench top study of the AC-coupled LMFE board.

\section{amorphous-Ge film}

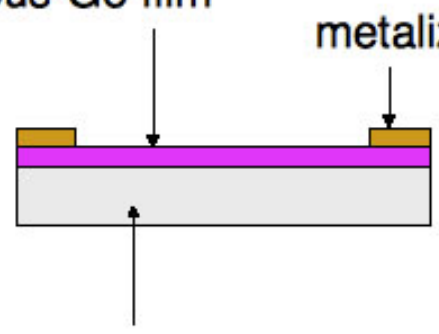

\section{substrate}

Figure 29: A schematic illustration for the LMFE prototype board
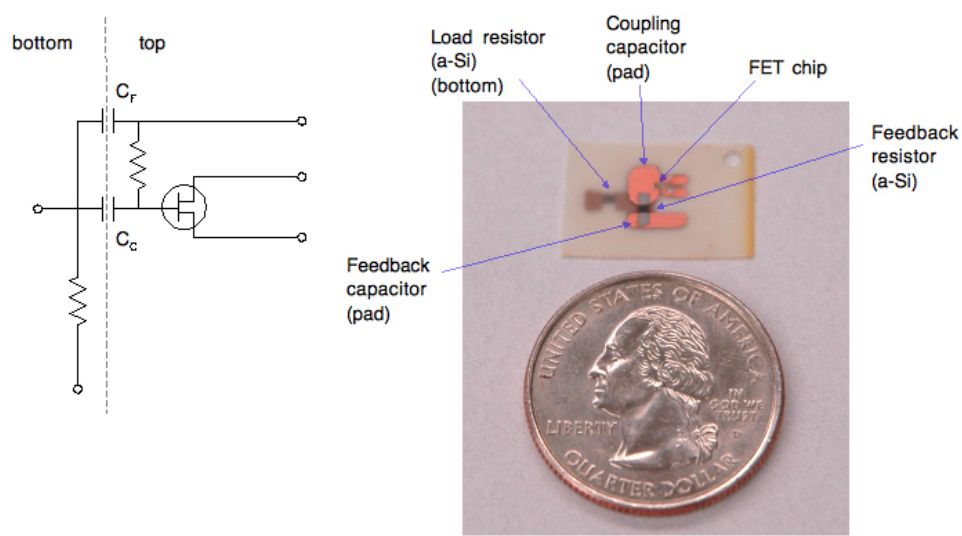

Figure 30: A fabricated AC-coupled Low Mass Front End (LMFE) board 


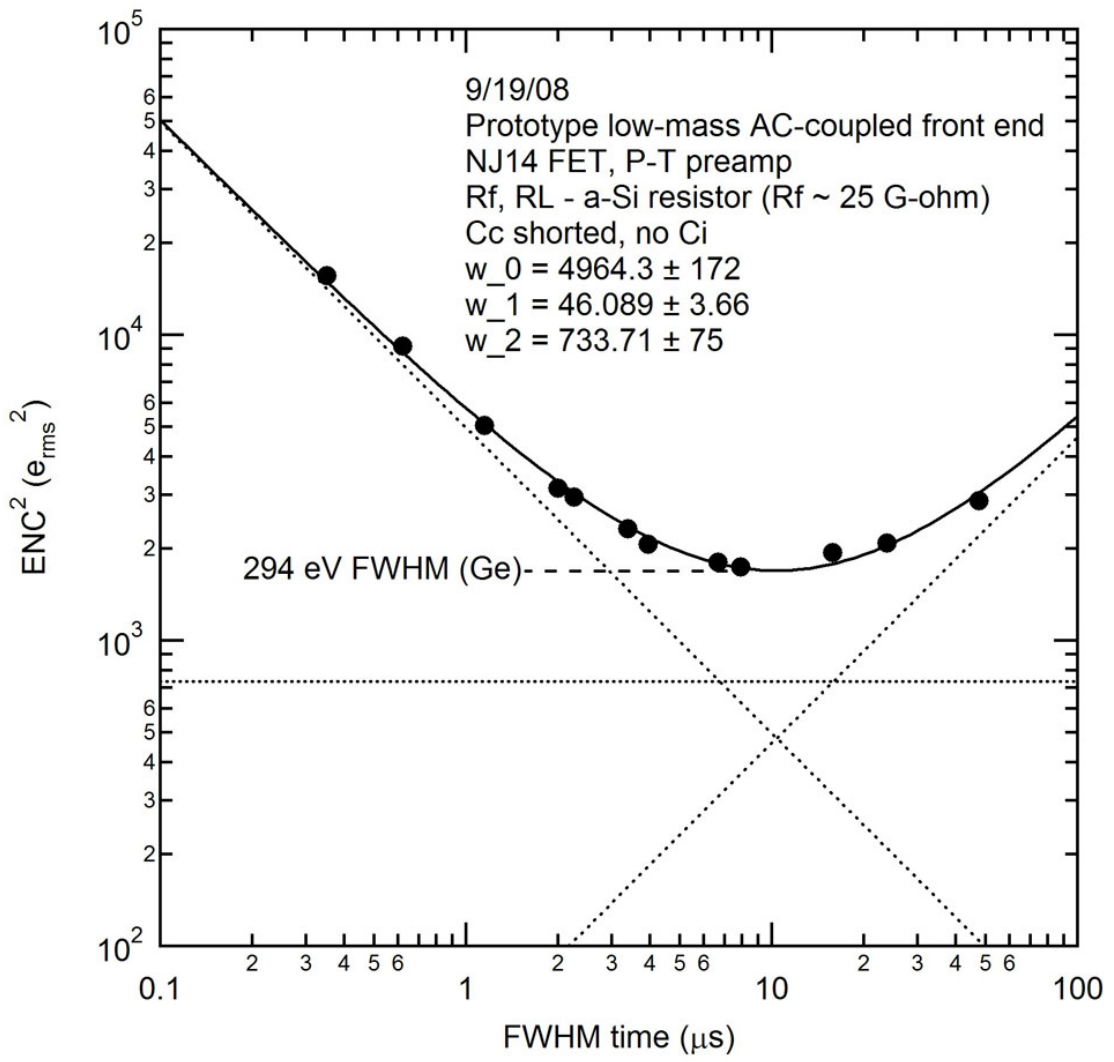

Figure 31: Room temperature noise measurement of the LMFE. 


\section{Material Screening Related to the Signal Path}

Several candidate materials for fabricating cables and electronics components were screened at the LBNL's Low Background Facility. This included employing neutron activation techniques by irradiating samples at the UC Davis Reactor followed by gamma counting. In addition, a few commercially available cables and preamplifier assemblies had been screened for their level of radioactivity.

\subsection{Substrate Material for the LMFE}

The two candidates for the substrate material we have considered are fused silica and sapphire. A batch of 10 fused silica wafer discs was purchased and screened with direct gamma counting. Only limits of the $\mathrm{U}$, Th, and $\mathrm{K}$ radioactivity were obtained due to the limiting small sample mass $(\sim 10 \mathrm{~g})$ (see Table 6). Also shown for comparison are values obtained for Suprasil quartz tubing.

Table 6: Substrate Material Screening

\begin{tabular}{|c|c|c|}
\hline & fused silica wafer & Suprasil tubing \\
\hline \hline $\mathrm{U}$ & $40 \mathrm{ppb}$ & $0.5 \mathrm{ppb}$ \\
\hline $\mathrm{Th}$ & $70 \mathrm{ppb}$ & $1 \mathrm{ppb}$ \\
\hline $\mathrm{K}$ & $90 \mathrm{ppm}$ & $0.4 \mathrm{ppm}$ \\
\hline
\end{tabular}

\subsection{New candidate material for cables - Parylene INAA at MNRC}

The stringent low background requirements of the MAJORANA Demonstrator R\&D Project call for structural materials of the utmost radiopurity, particularly with respect to the $\mathrm{U}$ and Th decay chains. For some materials, the radiopurity requirements are so strict, or the materials are so expensive to buy in sufficient quantities, that direct gamma counting is infeasible. Many such materials, in particular plastics, are assayable via instrumental neutron activation analysis (INAA), in which a small sample of the material is irradiated in a bath of thermal, epithermal, and/or fast neutrons (i.e. by placing the sample in or near a nuclear reactor core), and the produced radioisotopes identified and quantified by direct gamma counting. The knowledge of the incident neutron flux, usually obtained via the co-irradiation of a "standard", is then used to deduce the levels of the various impurities residing in the sample material.

The MAJORANA collaboration has direct access to several research reactors and counting facilities in which such work can be performed. One such pairing is the McClellan Nuclear Radiation Center (MNRC), associated with UC Davis and located in Sacramento, CA, and the LBNL counting facilities on the surface in Berkeley, CA and underground in Oroville, CA. These facilities have been employed in the past to perform INAA for $\mathrm{U}$ and $\mathrm{Th}$ in plastics for other low-background experiments, for example CUORE. Another such research reactor and counting facility is located at North Caroline State University. However, the NC State facility is inexperienced in performing INAA for U and Th in plastics.

The Majorana Materials and Assay working group proposed to perform INAA on a sample of parylene powder, a plastic of interest for the MAJORANA Demonstrator apparatus. In order to test the capabilities of the NC State facility while simultaneously assuring an accurate, sensitive count of the material, the group opted to send parylene samples to both NC State and MNRC/LBNL for irradiation and counting. This document reports on the results obtained from MNRC/LBNL. 


\subsubsection{Parylene Samples}

Parylene is a thin-film plastic commonly used as a coating and known for its good surface conformability and low dielectric constant [12. Several hundred grams of raw Parylene N and C powder was obtained from Para Tech Coating, Inc. by MAJORANA collaborators at the University of Washington. The powder was transferred under an Ar gas atmosphere into small polypropylene vials following leaching procedures provided by PNNL chemists [13]. The details of the parylene transfer is currently only available on the MAJORANA TWiki [14. The vials were then sent to NC State where they were sealed with soldering iron. Three of the vials were then shipped to LBNL for irradiation at MNRC. Details of the vials are listed in Table 7 The filling factor of the vials was kept near $\sim 50 \%$ to minimize containment failure in the event of large thermal expansion during irradiation.

Table 7: Details regarding the parylene samples sent to MNRC for irradiation.

\begin{tabular}{c|c|c|c|c}
\hline Vial No. & Size & Contents & Lot No. & Fill mass \\
\hline \hline 4 & $8.06 \mathrm{~mL}$ & Parylene N & 06N15002 & $3.19 \mathrm{~g}$ \\
9 & $1.40 \mathrm{~mL}$ & Parylene N & 06N15002 & $0.48 \mathrm{~g}$ \\
10 & $1.40 \mathrm{~mL}$ & Parylene N & 06N15002 & $0.53 \mathrm{~g}$ \\
\hline
\end{tabular}

\subsubsection{Ceramic Standard}

For in-situ calibration of the neutron fluence on the samples, a small ceramic standard was prepared containing known levels of ${ }^{238} \mathrm{U},{ }^{232} \mathrm{Th}$, and other elements. $51.25 \mathrm{mg}$ of such "standard pottery" was sealed into a $5 / 8$ " diameter aluminum disk using a bullet press.

\subsubsection{Irradiation at MNRC}

MNRC is home to a TRIGA ${ }^{\mathrm{TM}}$ reactor rated to $2 \mathrm{MW}$. MNRC provides an array of options for sample irradiations, including both in-core and out-of-core locations with varying encapsulation requirements, neutron spectra, and total $\mathrm{n}$ fluence limits. A picture of the reactor from above is shown in Figure 32. For this irradiation we opted to use MNRC's Pneumatic Transfer System, in which small samples are placed in a poly "rabbit" and transfered into and out of the reactor automatically via a pneumatic system. Consideration of the structural integrity of the rabbits limits irradiations in this in-core position to $<1 \mathrm{MWh}$.

The parylene samples and ceramic standard were hand-delivered to MNRC on June 9, 2008. The larger parylene vials was placed in the bottom of the rabbit, followed by the ceramic standard, and finally by the two smaller parylene vials. When inserted into the reactor, the rabbit was oriented upside-down relative to filling, so that gravity pulled the parylene in the larger vial as close as possible to the ceramic standard. The rabbit was irradiated at $1 \mathrm{MW}$ for 1 hour, corresponding to a total fluence of $\sim 2 \times 10^{16}$ thermal neutrons per $\mathrm{cm}^{2}$, and $\sim 9 \times 10^{15}$ fast neutrons per $\mathrm{cm}^{2}$. The irradiation changed the color of the rabbit from its initial translucent white to the chocolaty-brown color shown in Figure 33 .

After irradiation, the rabbit and samples were allowed to cool for $\sim 1$ day. After cooling, the rabbit was opened, and the parylene vials extracted and briefly counted. The samples were deemed cool enough to ship, and were sent to LBNL via next-day FedEx. The ceramic standard required several more days to cool down to levels safe for shipping. It was sent to LBNL via next-day FedEx on June 12, 2008.

\subsubsection{Sample Transfer}

Vials were received at LBNL by Radiation Technician Steve Sohner. Radiation Technician Jeff Bramble transferred the powder from the larger vial to a small plastic zipper storage bag under a 


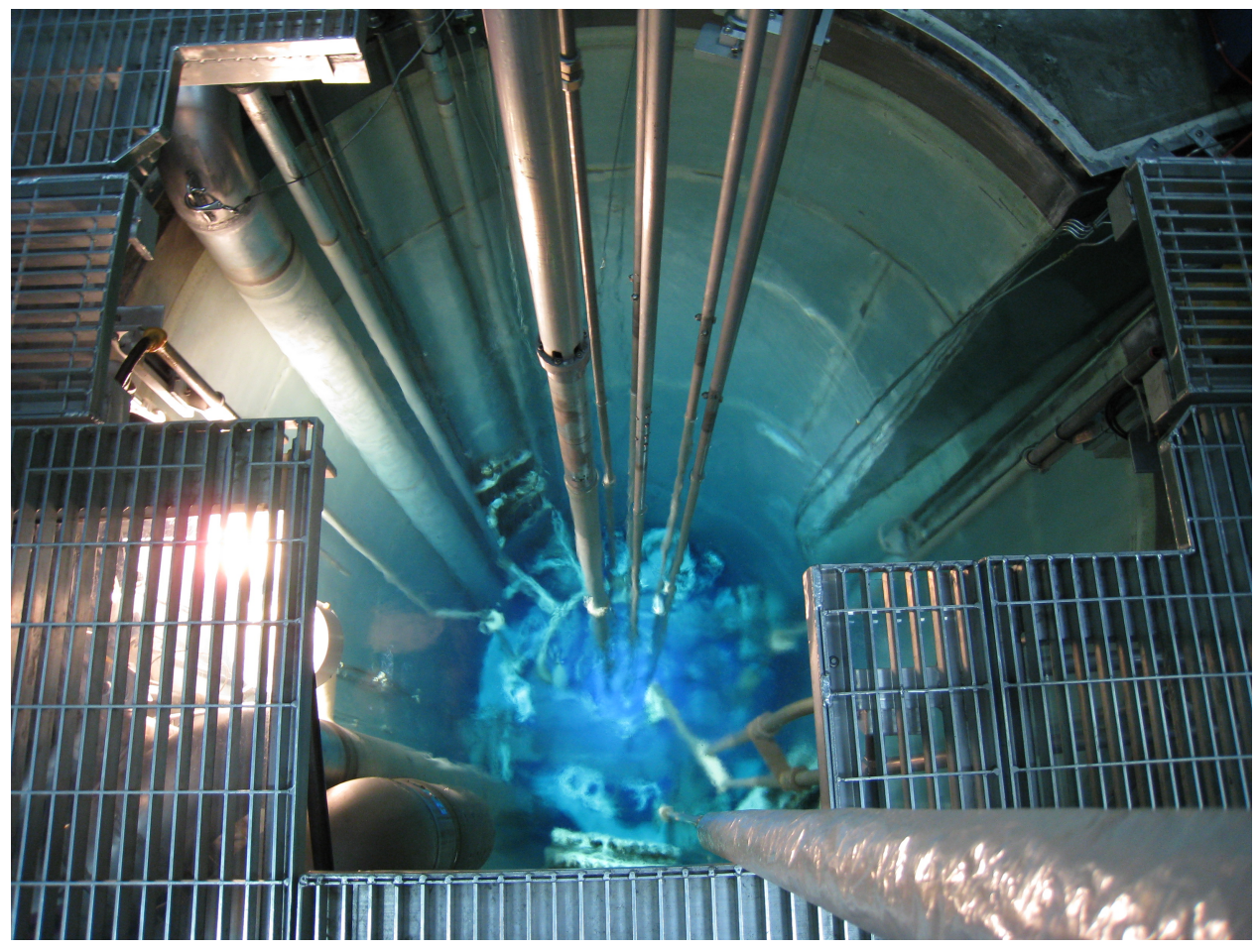

Figure 32: View of the MNRC TRIGA ${ }^{\mathrm{TM}}$ reactor from above.

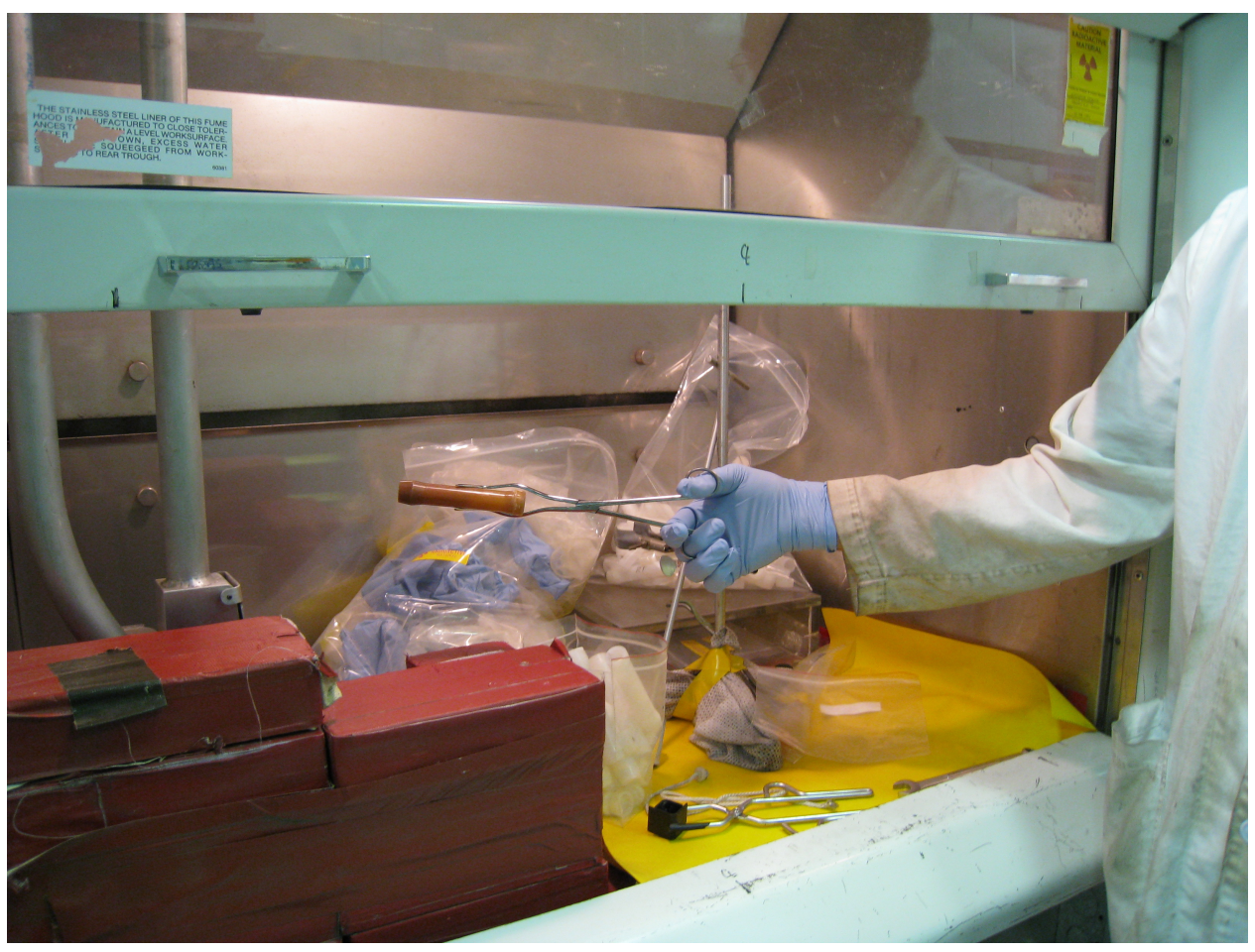

Figure 33: Irradiation turned the rabbit a chocolaty-brown color. 
hood. The vial was also placed in a separate bag. Both bags were placed in double containment and labeled; the final samples ready for counting are shown in Figure 34. The two smaller vials were deemed to have too little powder mass to make increase significantly the sensitivity of the measurement, and were placed into sample storage by Bramble. A similar procedure was followed for the ceramic standard when it arrived at LBNL on June 13, 2008.

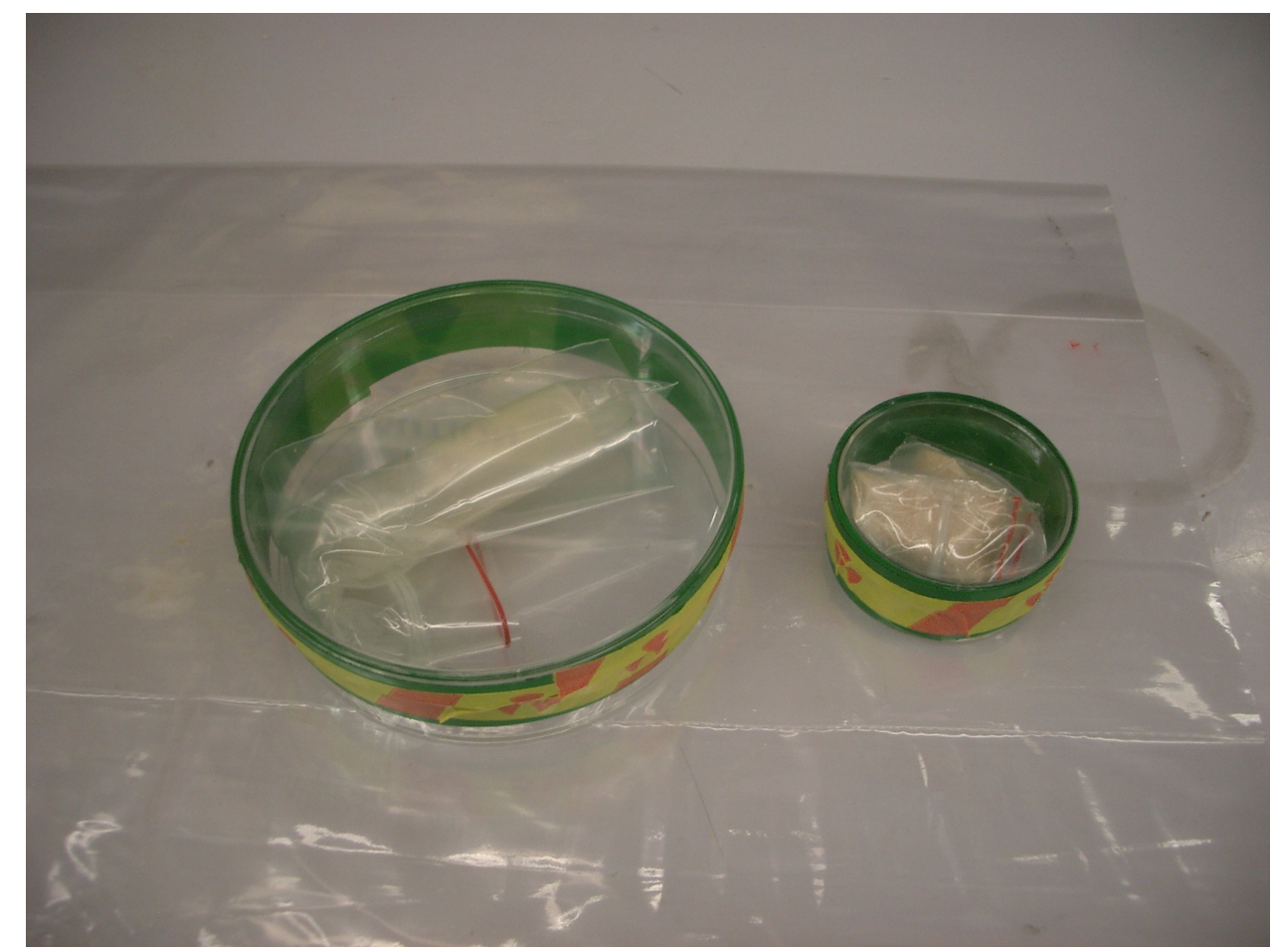

Figure 34: The parylene powder sample (right) and the polypropylene vial in which it was irradiated, bagged, double-contained, labeled, and ready for counting.

\subsection{Counting and Results}

The parylene and ceramic standard were repeated counted for $\sim 1$ month at the LBNL surface counting facility. The counting time for individual runs varied between 1 hour and 7 days. An example parylene spectrum, taken June 11, 2008 for 16 hours 40 minutes, is shown in Figure 35. The spectrum is dominated by ${ }^{82} \mathrm{Br}$ and ${ }^{24} \mathrm{Na}$; many of the unidentified lines in the figure are sum lines from these radioisotopes. These radioisotopes limit the sensitivity for identifying the 277 $\mathrm{keV}$ peak from ${ }^{239} \mathrm{~Np}$ that gives the level of ${ }^{238} \mathrm{U}$ in the sample. $\mathrm{Br}$ is commonly used in plastic manufacturing, and is likely resident in the sample itself. $\mathrm{Na}$ is a common residue left by human contact, although if this is the source of the ${ }^{24} \mathrm{Na}$ then it is unclear whether the sample transfer was insufficiently clean or if the sample as received from the manufacturer was already dirty. There is also a large line from ${ }^{198} \mathrm{Au}$, which is readily activated when even a small amount of $\mathrm{Au}$ is present. Au could be introduced to the sample via human contact (e.g. residue from a wedding ring) or from gold residue left on surfaces in the sample transfer room or in the leaching containers.

Similarly, at later times, the region surrounding the $312 \mathrm{keV}{ }^{233} \mathrm{~Pa}$ peak that is used to quantify the level of ${ }^{232} \mathrm{Th}$ is dominated by ${ }^{51} \mathrm{Cr}$ and ${ }^{65} \mathrm{Zn}$. Other isotopes identified by gamma counting include ${ }^{58} \mathrm{Co},{ }^{60} \mathrm{Co},{ }^{59} \mathrm{Fe},{ }^{140} \mathrm{La},{ }^{122} \mathrm{Sb},{ }^{124} \mathrm{Sb},{ }^{46} \mathrm{Sc},{ }^{47} \mathrm{Sc},{ }^{153} \mathrm{Sm}$, and potentially also ${ }^{99} \mathrm{Mo}$. These are radioisotopes found in common dirt. As for ${ }^{24} \mathrm{Na}$ and ${ }^{198} \mathrm{Au}$, it is unclear whether dirt could 


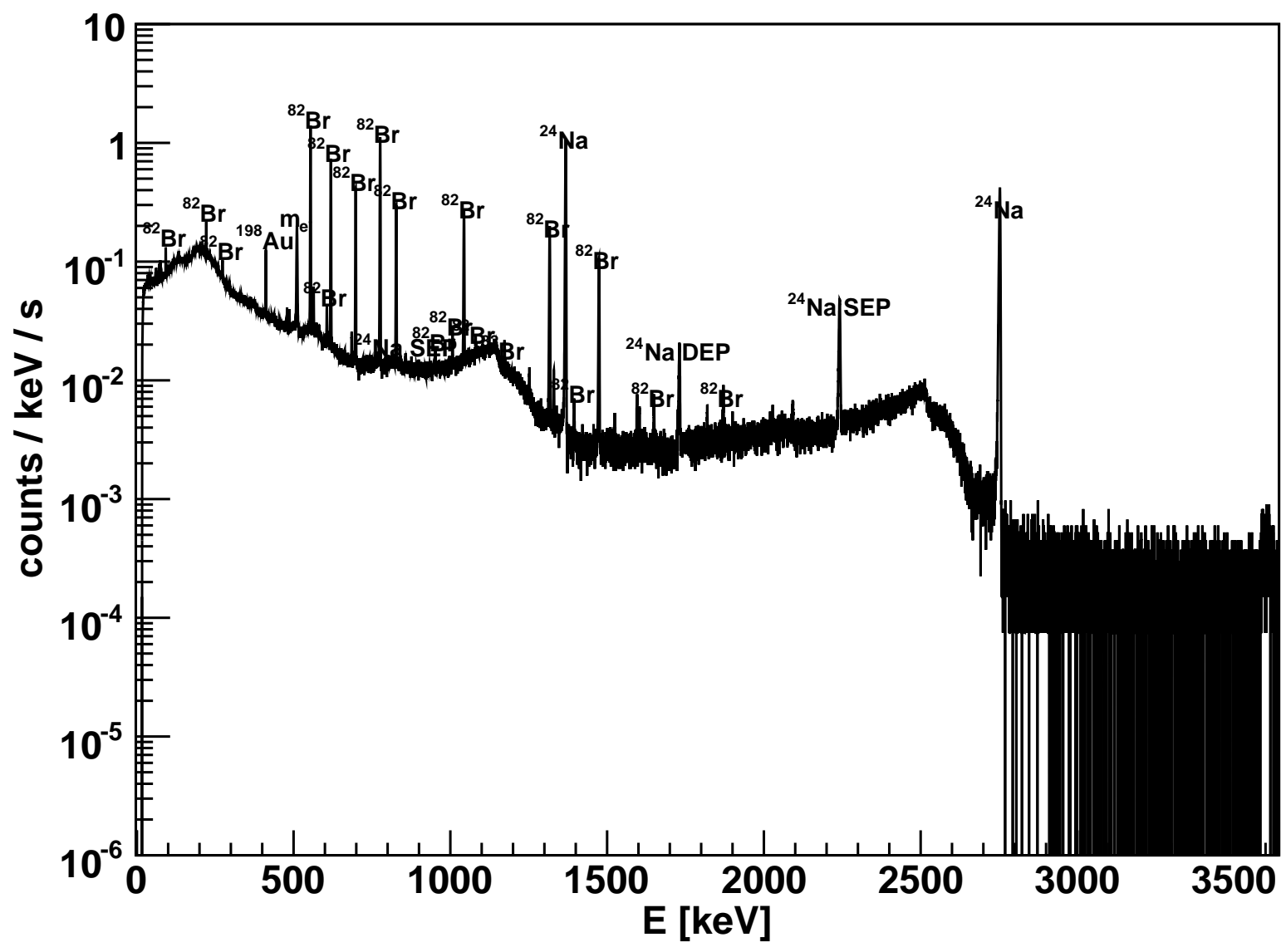

Figure 35: Gamma spectrum for parylene taken June 11, 2008. The livetime is 60000 seconds. 
have been introduced during sample preparation, or if it was already present in the sample when it was received from the manufacturer.

To set quantitative limits on the level of ${ }^{238} \mathrm{U}$, a 1200 minute count starting 5 days after irradiation was used. A consecutive count of the ceramic standard was used to normalize the counting rate in the region of the $277 \mathrm{keV}{ }^{239} \mathrm{~Np}$ peak. A small peak was visible in this region but it was statistically consistent with background. The upper limit on the concentration of ${ }^{238} \mathrm{U}$ in the parylene was determined to be $30 \mathrm{ppt}$.

For ${ }^{232} \mathrm{Th}$, a 4600 minute count starting $\sim 1$ month after irradiation was used along with a consecutive count of the ceramic standard. As for ${ }^{239} \mathrm{~Np}$ in the earlier count, a small but insignificant peak was visible in the region of the $312 \mathrm{keV}{ }^{233} \mathrm{~Pa}$ peak. From this, an upper limit of $50 \mathrm{ppt}$ was derived on the concentration of ${ }^{232} \mathrm{Th}$ in the parylene.

This task lasted a total of $\sim 6$ months from beginning to end. A vast majority of that time was spent determining the sample preparation protocols and irradition logistics. Powders are a particularly difficult material to subject to INAA, because they are not easy to clean or to keep clean, and they require special handling procedures post-irradiation. In addition, this was the first time our group has performed such work, and considerable effort was needed to make the right connections, to prepare and understand all procedures, to set up accounts to fund the work, and to ensure the work was performed safely and without error. Now that we are set up to perform this work, and all involved parties are up-to-speed on the work needed, future irradiations should take minimal planning effort, with most of the time spent during irradiation and sample counting.

\subsubsection{Potential Improvements}

It will be important to try to understand the sources of the various contaminants that limited our sensitivities for this sample. In particular, it would be a good idea to review the sample preparation procedure and identify points where $\mathrm{Na}, \mathrm{Au}$, or dirt could have been introduced. It will also be important in future samples to request the handling history prior to receipt from the manufacturer.

Significant sensitivity improvements can be achieved by simply irradiating larger samples for a longer period of time. Although the PTS used in this irradiation is limited to a $1 \mathrm{MWh}$ exposure, in conversations with the reactor operators we learned that a perhaps more suitable position would be an out-of-core position using sample holders made of Si. Although the total $\mathrm{n}$ flux in this position is more than an order-of-magnitude lower than in the PTS, it is much softer (dominated by thermal n) and does not have exposure limitations. MNRC has recently performed irradiations up to $14 \mathrm{MWh}$ in this position, and expressed in conversation that even longer radiations are not problematic from their perspective.

Finally, for Th measurements, in the case that we are limited by natural backgrounds present at the LBNL surface counting facility, an additional order of magnitude or so in sensitivity can be achieved by counting samples at the Oroville counting facility. Samples should always first be sent to LBNL for counting in the surface facility to determine whether the sensitivity is limited by the presence of other radioisotopes present in the material, as was the case for this sample of parylene. Samples passing this initial screening count can be transported to Oroville for a lower-background underground assay.

\subsection{Other samples}

A sample bundle of commercially available coaxial cable, and a batch of 32 vendor-assembled preamps (see Fig. 36) with choice electrical components (but not specified for low background application) were screened for radioactivity at the LBF. The latter forms a baseline for contamination studies of vendor fabrication processes. 


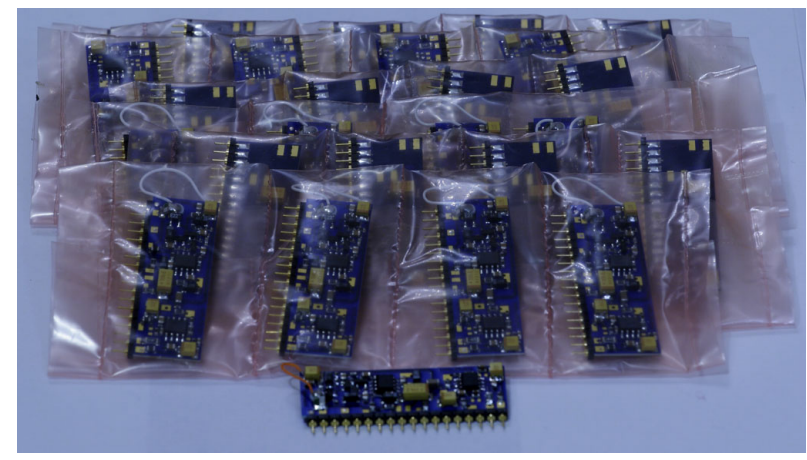

Figure 36: A batch of 32 assembled preamps screened for overall radioactivity.

\section{Summary and Conclusion}

A comprehensive study of the NSC signal path, including prototyping the critical main long flex-cable and the central channel AC-decoupling board, has been explored in the present work. Based on the R\&D work described above, we propose the below electronics configuration scheme for the segments and central contacts of the NSC detectors. The proposed scheme has taken into consideration several criteria - such as low mass, low background, good energy resolution, low crosstalk, low noise, and fast signal rise time - that are important for the MAJORANA project. The recommendations, however, rely on the successful sourcing of material that meets the radioactivity requirements of the experiment.

\subsection{Recommendation for the Segment Readout}

- Use Warm FETs outside the cryostat.

- Use a short cable (minimal mass and width) to reach the coldplate, followed by a long cable (signal transfer) across the coldplate and along the crossarm to the preamps. The longer cable could have a more open geometry to reduce signal crosstalk.

- Segment preamps should be outside the vacuum cryostat and shielded for radioactivity. Separation of the preamplifiers into multi-stage modules with the final stages positioned far away from the detector should be considered.

- Use low mass flexi-cables with copper traces on clean CuFlon/PEN dielectrics.

- Typical cable dimensions could be similar to those described in Section 3 .

- To compensate for the risetime, a preamplifier topology similar to the one described in 9 ] would be preferred.

\subsection{Recommendation for the Central Contact}

- Use a cold FET inside the vacuum and close to the crystal.

- Adopt the alternative AC-coupling approach for signal-to-HV decoupling.

- Use bare die FETs (wire bonded to circuit board) with feedback and coupling capacitors designed as part of the FET circuit board (e.g. the LMFE prototype).

- Use multi-trace flex-cable (e.g. CuFlon) to connect the FET circuit board to the rest of the amplifier.

- The rest of the amplifier should be assembled outside of the cryostat.

- Amplifier topology is preferred to be similar to the one described for the segments in case a risetime boost is also needed for the central contact signal. 


\section{References}

[1] The Majorana Proposal http://majorana.npl.washington.edu/

[2] Y.-D. Chan, "Plan for an N-type Multi-Segmented Demonstrator", presented at the MAJORANA Collaboration Meeting, Seattle, WA (August 2007).

[3] F.S. Goulding, D.A. Landis, P.N. Luke, N.W. Madden, D.F. Malone, P.H. Pehl, and A.R. Smith, Semiconductor Detectors and Double Beta Decay, LBL Report LBL-16682 (1983).

[4] http://www.klapdor-k.de/

[5] http://www.nu.to.infn.it/exp/all/igex/

[6] http://www.mpi-hd.mpg.de/gerda/

[7] http://www-nsd.lbl.gov/programs/GRETINA.html

[8] http://www-win.gsi.de/agata/overview.htm

[9] L. Fabris, N.W. Madden, H. Yaver, A fast, compact solution for low noise charge preampliers, Nuclear Instruments and Methods in Physics Research, A 424 (1999) 545-551.

[10] V. Radeka, Signal, noise and resolution in position-sensitive detectors, IEEE Trans. Nuclear Science, NS-21, No. 1, Feb 74, 51-64

[11] J. Harder, V. Radeka, G. Smith, B. Yu, A low-noise, low stored energy preamplifier for highvoltage radiation detectors, Nuclear Instruments and Methods in Physics Research A 576 (2007) $397-402$

[12] Para Tech Coating, Inc. website, http://www.parylene.com, accessed July 8, 2009.

[13] E. Hoppe, "PNNL Plastics Leaching Procedure", M-TECHDOCDET-2008-016.

[14] Parylene topic on the Majorana TWiki, http://mjwiki.npl.washington.edu/bin/view/Majorana/Parylene?rev=13, accessed July 8, 2009 . 